\title{
Electrostatics of two-dimensional structures: exact solutions and approximate methods
}

\author{
Michael M. Fogler \\ Department of Physics, University of California San Diego, La Jolla, California 92093
}

(Dated: October 31, 2018)

\begin{abstract}
We consider a set of electrostatic problems relevant for determining the real-space structure and the ground-state energy of a two-dimensional electron liquid subject to smooth external potentials. Three fundamental geometries are investigated: an elongated metallic island, an antidot, and a constriction. In the first two cases complete closed-form analytical solutions are obtained, despite the absence of rotational or translational symmetries. These solutions govern the shape and size of large quantum dots, and also the size of the depletion regions and the density profiles around isolated antidots. For the constriction, an exact asymptotical formula for boundary shape is derived and arguments are given in favor of its universality. For the cases where the full analytical solution cannot be obtained, an approximate method is proposed as an alternative. Its accuracy is verified against numerical simulations in a periodic (checkerboard) geometry.
\end{abstract}

\section{INTRODUCTION}

\section{A. Formulation of the problem}

Studies of physical phenomena in two-dimensional metals and thin films often lead to mixed electrostatic boundary-value problems $\stackrel{1}{-1}$ A prototypical example is the task of determining the density profile $n(\mathbf{r})$ of a twodimensional (2D) electron liquid in the proximity of external charges or voltage sources. Such charges and sources are used in practical applications to manipulate the electron liquid into desired geometrical shapes, e.g., quantum dots, narrow wires, constrictions, etc. They are also used to intentionally introduce defects, such as antidots, 2.3 .4 .5 into an otherwise homogeneous system. In addition to artificial sources of external potentials, in real materials electrons also experience a random potential of ubiquitous charged impurities. It appears therefore that methods that can tackle the corresponding electrostatic problems could be of considerable value both for applied and for fundamental research. Unfortunately, the boundary-value problems are notoriously difficult to solve analytically. The goal of the this paper is to show that either the complete analytical solutions or their exact asymptotics can be found for a number of nontrivial basic geometries.

Our most unexpected unexpected result concerns the electrostatics of a constriction. We show in Sec. IV] that the long-range Coulomb interaction can cause a significant narrowing of the region occupied by the $2 \mathrm{D}$ electron liquid in the constriction and that the boundaries of this region are described by an interesting nonanalytic function, see Eq. (73).

The present article extends and generalizes the results available in the literature, e.g., Refs. 1.23 and 67 7 8,910 , and came as an outgrowth of our recent work ${ }^{11}$ on the electrostatics of disordered 2D systems.

We will consider 2D electron systems that are separated from grounded electrodes or other screening bodies by distances much larger than the interelectron spac- ing. Such conditions are realized in semiconductor heterostructures and in field-effect transistors with thick insulator layers. In these systems electrons interact via the $1 / r$ Coulomb law and form a metallic liquid if their density is not too low.

The results of the present work are obtained within the approximation that the Thomas-Fermi screening radius $r_{\mathrm{TF}}$ of the electron liquid metallic is vanishingly small. This is the correct leading-order approximation if all lengthscales of interest exceed $r_{\mathrm{TF}}$.

In typical semiconductor realizations of $2 \mathrm{D}$ systems, $r_{\mathrm{TF}}$ is of the order of the interelectron separation $a_{\mathrm{e}-\mathrm{e}}=$ $n^{-1 / 2}$, and so our approach is good for studying variations of $n(\mathbf{r})$ on lengthscales larger than $a_{\mathrm{e}-\mathrm{e}}$. Note that this does not necessarily prohibit us from describing some effects that are due to discreteness of electrons. For example, in Sec. III we will be able to calculate the energy spacing of the Coulomb blockade peaks because in the leading order it is determined by the classical capacitance. On the other hand, the equations below cannot be used, e.g., to study quantum dots with just a few electrons. Also, these equations do not apply too close to the edges of the metallic regions where $n(\mathbf{r}) \rightarrow 0$ and so, formally, $a_{\mathrm{e}-\mathrm{e}} \rightarrow \infty$. Still, even in these situations the solution of the corresponding electrostatic problem should provide a valuable insight.

In the approximation of vanishingly small $r_{\mathrm{TF}}$, the electrostatic potential $\Phi(\mathbf{r})$ in the regions occupied by the electron liquid is perfectly flat,

$$
\text { Metal: } n(\mathbf{r})>0, \quad e \Phi(\mathbf{r})=\mu_{e}=\text { const, }
$$

where $\mu_{e}$ is the electrochemical potential. The rest of the $2 \mathrm{D}$ plane is occupied by the depletion regions (DR) the areas of exponentially small, effectively zero electron density that are classically forbidden for the electrons:

$$
\text { DR: } \quad n(\mathbf{r})=0, \quad e \Phi(\mathbf{r})>\mu_{e} .
$$

Our goal is to study the conditions that cause DRs to appear and their detailed stricture 12 To finalize the formulation of the electrostatic problem we wish to solve for 
this purpose, we need an expression for $\Phi$ in terms of $n$. We distinguish two cases.

Case A.- If the number of electrons $N_{e}$ in the system is finite, we use

$$
\Phi(\mathbf{r})=\Phi_{e x t}(\mathbf{r})+\frac{e}{\kappa} \int d^{2} r^{\prime} \frac{n\left(\mathbf{r}^{\prime}\right)}{\left|\mathbf{r}-\mathbf{r}^{\prime}\right|},
$$

where $\Phi_{\text {ext }}(\mathbf{r})$ is the external electrostatic potential and $\kappa$ is the dielectric constant of the medium.

Case B. - If we deal with an infinite system with a nonzero average electron concentration, we define $\Phi$ as follows:

$$
\Phi(\mathbf{r})=\frac{e}{\kappa} \int d^{2} r^{\prime} \frac{n\left(\mathbf{r}^{\prime}\right)-\sigma\left(\mathbf{r}^{\prime}\right)}{\left|\mathbf{r}-\mathbf{r}^{\prime}\right|} .
$$

Here $\sigma(\mathbf{r})$ represents a spatially nonuniform background of opposite charge. In this formulation the primary parameter is the average background density $n_{e}=\langle\sigma(\mathbf{r})\rangle$, and the electrochemical potential in Eqs. (10) and (2) is determined by electroneutrality of the system as a whole, so that $\mu_{e}$ is a function of $n_{e}$. There is no loss in generality in assuming that the background charge density $\sigma(\mathbf{r})$ is confined to the same 2D plane as the electrons. Indeed, it is easy to see that an arbitrary three-dimensional charge density distribution $\sigma_{3}(\mathbf{r}, z)$ creates essentially the same electrostatic potential in the $z=0$ plane as the following effective 2D density:

$$
\sigma(\mathbf{r})=\int d^{2} r^{\prime} \int \frac{d z|z| \sigma_{3}\left(\mathbf{r}^{\prime}, z\right)}{2 \pi\left[\left(\mathbf{r}-\mathbf{r}^{\prime}\right)^{2}+z^{2}\right]^{3 / 2}} .
$$

The only difference between the two potentials is the $\mathbf{r}$ independent term $e^{2} C_{0}^{-1} n_{e}$, where $C_{0}^{-1}$, given by

$$
C_{0}^{-1}=\frac{4 \pi}{\kappa} \frac{\left\langle\sigma_{3}|z|\right\rangle}{\left\langle\sigma_{3}\right\rangle}
$$

is the inverse geometric capacitance per unit area between the $2 \mathrm{D}$ layer and the external sources $[\langle\ldots\rangle$ denotes the three-dimensional (3D) spatial average]. As the name implies, $C_{0}^{-1}$ is usually determined by the fixed dimensions of the structure and the electrodes, and so it is almost independent of $n_{e}$. In this situation, a finite $C_{0}^{-1}$ causes only a trivial linear shift of the electrochemical potential $\mu_{e}$, while the interesting quantity is the deviation

$$
\delta \mu \equiv \mu_{e}-e^{2} C_{0}^{-1} n_{e} .
$$

This is one of the quantities we will be calculating below. We will assume that $\Phi_{\text {ext }}(\mathbf{r})$ and $\sigma(\mathbf{r})$, whichever is appropriate, are smooth and bounded functions.

The major difficulty in solving the above equations stems from the mixed boundary conditions (11) and (2). These conditions do not specify where the boundaries of the DRs reside or what the potential inside the DRs is. They merely state that the DRs may exist and that inside of them $e \Phi(\mathbf{r})$ must exceed a certain constant value $\mu_{e}$. It seems that in the general case, one can make only the following two trivial statements. First, in the formulation (3) the metallic regions are located in areas where the potential energy $e \Phi_{\text {ext }}(\mathbf{r})$ is sufficiently low, the rest of the 2D plane being a DR. Second, in the formulation (4), DRs surround negative local minima of $\sigma(\mathbf{r})$. [If $\sigma$ is nonegative everywhere, then the sought ground state is simply $n(\mathbf{r})=\sigma(\mathbf{r})$ and the system is free of DRs].

As alluded to above, there has been a sizeable amount of work devoted to the electrostatics of disordered electron systems. Prominent early investigations include those of Efros and Shklovskii 13 on the nonlinear screening in 3D doped semiconductors and its extensions to 2D $\stackrel{14.15 .16}{1 n}$ disordered systems DRs typically have some irregular shapes, and so there is no any convenient coordinate system that can be used to take advantage of the known techniques of solving integral equations. Thus, the random case seems mathematically intractable. Rigorous results that have been obtained for such problems are limited to certain scaling laws 14.15 .16 and some asymptotical limits $\frac{11.16}{1}$ There is a hope however that in regular geometries analytical solutions could be easier to derive. This is indeed the case. For example, if the external potential $\Phi_{\text {ext }}(x, y)$ depends only on one coordinate, say, $x$, then the problem can be reduced to an equation for just a few parameters, the positions of the DR edges 17 Once they are determined, the density $n(x)$ at all other $x$ can be found from simple analytical formulas, see an example below. Similarly, there is a closed-form solution in quadratures for the axially-symmetric case, $\Phi_{\text {ext }}=\Phi_{\text {ext }}(r)$, provided there is only one DR ${ }^{1}$ In other words, it is known how to find the density profile $n(r)$ of a single round droplet or around a circular depletion hole. Let us give a few examples.

\section{B. Examples of exact solutions}

Example 1.- Our first example is a metallic droplet confined laterally by a parabolic external potential

$$
e \Phi_{e x t}(r)=\frac{1}{2} U_{x x} r^{2}, \quad U_{x x}>0 .
$$

The density profile of such a droplet is known to be hemispherical1

$$
n(r)=\frac{2}{\pi^{2}} \frac{\kappa}{e^{2}} U_{x x} \sqrt{a^{2}-r^{2}},
$$

with the radius $a$ of the droplet related to its electrochemical potential by $\mu_{e}=U_{x x} a^{2}$.

Example 2.- Another instructive example is an isolated DR in the form of a perfect circle. As noted above, such a depletion hole can form when $\sigma(\mathbf{r})$ has an isolated negative minimum, $\sigma_{0}<0$. Let this minimum be located at the origin, $r=0$, and let the radius $a$ of the induced depletion hole be small enough so that the expansion

$$
\sigma(\mathbf{r})=\sigma_{0}+\frac{1}{2} \sigma_{x x} r^{2}
$$


can be used. Following the formalism of Ref. 1 it is easy to find that

$$
a^{2}=-3 \sigma_{0} / \sigma_{x x},
$$

so that $n(r)=0$ at $r \leq a$. At $r>a, n(r)$ takes the form 11

$$
n(r)=\frac{\sigma_{x x} a^{2}}{\pi}\left[\sqrt{\frac{r^{2}}{a^{2}}-1}+\left(\frac{r^{2}}{a^{2}}-\frac{2}{3}\right) \arccos \frac{a}{r}\right] .
$$

Example 3.- Our last example is the case of a $y$ independent background density

$$
\sigma(\mathbf{r})=\sigma(x)=\sigma_{0}+\frac{1}{2} \sigma_{x x} x^{2},
$$

which gives rise to the DR in a form of a stripe of width $2 a$ flanked by the density distribution ${ }^{17}$

$$
\begin{aligned}
n(\mathbf{r}) & =\frac{1}{2} \sigma_{x x}|x| \sqrt{x^{2}-a^{2}}, \\
a^{2} & =-4 \sigma_{0} / \sigma_{x x}
\end{aligned}
$$

on the two sides. Note that all the presented exact solutions, Eqs. (9), (12), and (14), agree with a well-known result $\frac{18}{}$ that $n(\mathbf{r})$ has a square-root singularity near the edge of the DR. Previously, these solutions and their generalizations have been used to study the edges of $2 \mathrm{D}$ electron liquid,,$\frac{7}{?}$ quantum wires,$\frac{8.9}{,}$ quantum dots,$\frac{9}{=}$ and antidots 2.3

In a sense, all the aforementioned examples are onedimensional because $\Phi_{e x t}(\mathbf{r})$ [or $\sigma(\mathbf{r})$ ] depend on a single variable. To the best of our knowledge, there are no published exact solutions for truly 2D cases, i.e., for the geometries where $\Phi_{\text {ext }}(\mathbf{r})$ and $\sigma(\mathbf{r})$ are smooth functions of position and have no translational or rotational symmetries. The present paper is aimed to fill this gap. The geometries we consider are as follows. In Sec. II we study a droplet in a parabolic but not necessarily axially-symmetric confining potential. We show that the droplet has the elliptic shape, with Eq. (9) recovered as a special case. In Sec. III we derive a formula for the density profile around an elliptic depletion hole. This formula bridges the limiting cases of Eqs. (12) and (14). In Sec. IV we treat the nonlinear screening problem for the saddle-point, which is presumably the most interesting basic geometry. We derive the asymptotical formula for the width of the constriction, Eq. (73), and give arguments in favor of its universality. In Sec. $\nabla$ we examine the periodic ("checkerboard") external potential, which attains two goals. First, it enables us to study the interplay of the three fundamental geometries (dot, antidot, and the saddle-point) examined in Secs. IIIV Second, it serves as a testground for an approximate method of solving electrostatic problems suggested in our previous publication. ${ }^{11}$

At the end of each of the following Sections we briefly comment on the relevance of the obtained results for various types of experiments. A detailed comparison with the available experimental data is deferred for future work.

\section{ELLIPTIC ISLAND}

In this Section we derive the density profile of a metallic droplet that resides in the external potential

$$
e \Phi_{e x t}(\mathbf{r})=\frac{1}{2} U_{x x} x^{2}+\frac{1}{2} U_{y y} y^{2}, \quad 0<U_{x x} \leq U_{y y} .
$$

If $U_{x x}=U_{y y}$ we must recover Eq. (9); otherwise, if $U_{x x}<$ $U_{y y}$, we expect the droplet to be stretched out in the $x$ direction, along which the confinement is softer.

The quickest way to obtain the solution for arbitrary $U_{x x}$ and $U_{y y}$ is to use a classic theorem of Frank W. Dyson ${ }^{20}$ One of the corollaries of this theorem concerns the $2 \mathrm{D}$ charge density distribution

$$
n(x, y)=n_{d} \sqrt{1-\frac{x^{2}}{a^{2}}-\frac{y^{2}}{b^{2}}},
$$

which defines an elliptically shaped droplet with semiaxes $a$ and $b$. The Dyson theorem indicates that such a droplet creates the in-plane electrostatic potential of the form

$$
\begin{aligned}
\Phi_{d}(x, y) & =(e / \kappa) \pi n_{d} \int_{\lambda}^{\infty} \frac{a b d l}{\sqrt{\left(a^{2}+l^{2}\right)\left(b^{2}+l^{2}\right)}} \\
& \times\left(1-\frac{x^{2}}{a^{2}+l^{2}}-\frac{y^{2}}{b^{2}+l^{2}}\right),
\end{aligned}
$$

where $\lambda$ is equal to zero inside the droplet and is equal to the largest root of the equation

$$
\frac{x^{2}}{a^{2}+\lambda^{2}}+\frac{y^{2}}{b^{2}+\lambda^{2}}=1
$$

otherwise. This statement can be proved by expanding $\Phi_{d}(\mathbf{r})$ in ellipsoidal harmonics $\stackrel{21}{\stackrel{ }{a}}$ see Sec. IIII and App. A

Performing the integration in Eq. (18) for the case $\lambda=$ 0 , we get

$$
\Phi_{d}=\frac{\pi e b n_{d}}{\kappa}\left[K-\frac{K-E}{k_{d}^{2}} \frac{x^{2}}{a^{2}}-\frac{E-\left(1-k_{d}^{2}\right) K}{k_{d}^{2}} \frac{y^{2}}{b^{2}}\right] .
$$

Here $K$ and $E$ are the complete elliptic integrals of the first and the second kind, respectively, ${ }^{22}$ evaluated at

$$
k_{d}=\sqrt{1-(b / a)^{2}} .
$$

(As explained above, we expect $a \geq b$ ). Equations (16) and (20) indicate that if we choose $a, b$, and $n_{d}$ appropriately, we can satisfy the equilibrium condition (11). Indeed, we have $e \Phi=e \Phi_{e x t}+e \Phi_{d}=\mu_{e}$ in the interior of the droplet if the following equations hold:

$$
\begin{aligned}
\frac{U_{x x}}{U_{y y}} & =\frac{\left(1-k_{d}^{2}\right)\left[K\left(k_{d}\right)-E\left(k_{d}\right)\right]}{E\left(k_{d}\right)-\left(1-k_{d}^{2}\right) K\left(k_{d}\right)}, \\
\frac{a^{2}}{\mu_{e}} & =\frac{2}{U_{x x}} \frac{K\left(k_{d}\right)-E\left(k_{d}\right)}{K\left(k_{d}\right) k_{d}^{2}}, \\
\frac{n_{d}^{2}}{\mu_{e}} & =\frac{\kappa^{2}}{2 \pi^{2} e^{4}} \frac{U_{x x} k_{d}^{2}}{\left(1-k_{d}^{2}\right)(K-E) K} .
\end{aligned}
$$


It is easy to see that these equations have a unique solution for $a, b$, and $n_{d}$. Also, from the fact that the integrand in Eq. (18) is nonnegative, we conclude $\Phi_{d}(\mathbf{r})$ decreases with $r$, which ensures that the inequality (2) is also satisfied. Thus, Eq. (17) is the desired solution.

Although the parameters of this solution cannot be expressed in terms of elementary functions, one can work out the limiting cases, which are as follows. Let us define

$$
z_{d} \equiv U_{y y} / U_{x x} \geq 1
$$

If $z_{d}=1-\delta z$, where $\delta z \ll 1$, we have a nearly circular droplet with the following parameters:

$$
\begin{aligned}
a^{2} & =\frac{\mu_{e}}{\sqrt{U_{x x} U_{y y}}}\left[1+\frac{2}{3} \delta z+O\left(\delta z^{2}\right)\right], \\
b^{2} & =\frac{\mu_{e}}{\sqrt{U_{x x} U_{y y}}}\left[1-\frac{2}{3} \delta z+O\left(\delta z^{2}\right)\right], \\
n_{d} & =\frac{2}{\pi^{2}} \frac{\kappa}{e^{2}}\left(U_{x x} U_{y y}\right)^{1 / 4} \mu_{e}^{1 / 2}+O\left(\delta z^{2}\right) .
\end{aligned}
$$

In the limit $\delta z \rightarrow 0$, we indeed recover Eq. (9). In the opposite limit, $z_{d} \gg 1$, we have a strongly elongated droplet with parameters

$$
\begin{aligned}
a^{2} & =\frac{2 \mu_{e}}{U_{x x}}\left[1-\frac{2}{\mathcal{L}}+O\left(\frac{1}{\mathcal{L}^{2}}\right)\right], \quad \mathcal{L} \equiv \ln z_{d}, \\
b^{2} & =\frac{4 \mu_{e}}{U_{y y}}\left[\frac{1}{\mathcal{L}}+O\left(\frac{1}{\mathcal{L}^{2}}\right)\right] \\
n_{d} & \simeq \frac{1}{2 \pi} \frac{\kappa}{e^{2}} U_{y y} b\left(1+\frac{1}{2 z_{d}}\right) .
\end{aligned}
$$

Note that by setting $z_{d}$ to infinity and $x$ to zero, we obtain the solution for another geometry of basic interest: an infinite wire in the parabolic confining potential $e \Phi_{e x t}(y)=U_{y y} y^{2} / 2$. The density distribution in a crosssection of such a wire is a semicircle,$\underline{6}$

$$
n(y)=\frac{\kappa}{2 \pi e^{2}} U_{y y} \sqrt{b^{2}-y^{2}},
$$

see Eqs. (17) and (31).

Finally, let us calculate the capacitance of the droplet, $C_{d}=e^{2} d N_{e} / d \mu_{e}$, where $N_{e}$ total number of electrons in the droplet. Integrating $n$ in Eq. (17) over the area, we get $N_{e}=(2 \pi / 3) n_{d} a b$. Using this result, Eqs. (22)-(24), and some simple algebra, we find

$$
C_{d}=\frac{\kappa}{K\left(k_{d}\right)} a .
$$

As expected, the capacitance scales linearly with the linear size $a$ of the droplet.

One application of the derived results is the formula for the energy separation $e \Delta V_{g}$ of the Coulomb blockade ${ }^{23}$ peaks that would be observed if the droplet is weakly connected to external leads. Here $V_{g}$ has the physical meaning of the voltage on a gate that controls the size of the droplet, with conversion factor appropriate for the particular experimental geometry included. In the first approximation, $23=\Delta V_{g} \simeq e^{2} / C_{d}$. The analytical asymptotics of this expression are as follows:

$$
\begin{aligned}
\left(e \Delta V_{g}\right)^{3} & \simeq \frac{\pi^{2}}{6} \frac{e^{4}}{\kappa^{2}} \frac{\sqrt{U_{x x} U_{y y}}}{N_{e}}, \quad U_{y y} \simeq U_{x x}, \\
& \simeq \frac{1}{12} \frac{e^{4}}{\kappa^{2}} \frac{U_{x x}}{N_{e}} \ln ^{2} \frac{U_{y y}}{U_{x x}}, \quad U_{y y} \gg U_{x x} .
\end{aligned}
$$

Thus, for the droplet in a parabolic confinement, the separation between the Coulomb blockade peaks should scale as $N_{e}^{-1 / 3}$, with a coefficient of proportionality that depends on the asymmetry of the confining potential. This can be tested in experiments where both the size and the shape of the quantum dots can be controlled to some degree independently and the extensive statistics of the Coulomb blockade spacings can be accumulated, see, e.g., Ref. 24. A cautionary note is that one should study large dots, $N_{e} \gg 1$, where neglected here effects of disorder, single-particle level spacing, or subtle features of the edge structure ${ }^{25}$ (see also Ref. 26) are minimal.

\section{ELLIPTIC HOLE}

In this Section we consider the depletion hole that forms in a metallic 2D liquid around an isolated negative minimum of the background charge density $\sigma(\mathbf{r})$. This problem is relevant for, e.g., determining the density profile of the electron liquid around an antidot.

We assume that the minimum of $\sigma(\mathbf{r})$ is located at $r=0$ and that the depletion hole is small enough so that the expansion

$$
\sigma(\mathbf{r})=\sigma_{0}+\frac{1}{2} \sigma_{x x} x^{2}+\frac{1}{2} \sigma_{y y} y^{2}, \quad 0<\sigma_{x x} \leq \sigma_{y y},
$$

can be used $\left(\sigma_{0}<0\right)$. If $\sigma_{x x}=\sigma_{y y}$, then DR is a circle and Eq. (12) must be recovered; otherwise, if $\sigma_{x x}<\sigma_{y y}$, we expect the DR to be elongated in the $x$-direction, along which $\sigma(\mathbf{r})$ has the slowest growth. Indeed, below we show that the DR is the ellipse

$$
S: \frac{x^{2}}{a^{2}}+\frac{y^{2}}{b^{2}} \leq 1, \quad a \geq b .
$$

Our solution is based on the following series expansion of the three-dimensional electrostatic potential $\Phi(\mathbf{r}, z)$ :

$$
\Phi(\mathbf{r}, z)=\sum_{m=0}^{\infty} \sum_{\{p\}} \alpha_{m}^{p} F_{m}^{p}(\lambda) E_{m}^{p}(\mu) E_{m}^{p}(\nu),
$$

where $\lambda, \mu$, and $\nu$ are the ellipsoidal coordinates, $\frac{1.27}{1.27}$

$$
0 \leq \nu^{2} \leq k_{h}^{2} \equiv 1-\frac{b^{2}}{a^{2}} \leq \mu^{2} \leq 1 \leq \lambda^{2}
$$

that are related to the original Cartesian coordinates $x$, $y$, and $z$ by 


$$
x^{2}=\frac{a^{2}}{k_{h}^{2}} \lambda^{2} \mu^{2} \nu^{2}, \quad y^{2}=\frac{a^{2}}{k_{h}^{2}\left(1-k_{h}^{2}\right)}\left(\lambda^{2}-k_{h}^{2}\right)\left(\mu^{2}-k_{h}^{2}\right)\left(k_{h}^{2}-\nu^{2}\right), \quad z^{2}=\frac{a^{2}}{1-k_{h}^{2}}\left(\lambda^{2}-1\right)\left(1-\mu^{2}\right)\left(1-\nu^{2}\right) .
$$

The numerical coefficients $\alpha_{m}^{p}$ in Eq. (38) are to be determined, $p$ are certain real numbers (eigenvalues) that depend on $m$ and $k_{h}$, and $E_{m}^{p}(\xi), F_{m}^{p}(\lambda)$ are the ellipsoidal harmonics of the first and the second kinds $\underset{27}{\longrightarrow}$ respectively. The definition and some properties of $E_{m}^{p}$ and $F_{m}^{p}$ are reviewed in App. $\mathrm{A}$

Below we show that only the following $E_{m}^{p}$ harmonics 27 are present in the expansion (38):

$$
\begin{aligned}
& E_{1}^{p_{1}}(\xi)=\sqrt{1-\xi^{2}} \\
& E_{3}^{r_{ \pm}}(\xi)=\sqrt{1-\xi^{2}}\left(\xi^{2}-C_{ \pm}\right), \quad C_{ \pm}=\frac{1}{5}\left(1+2 k_{h}^{2} \mp \sqrt{1-k_{h}^{2}+4 k_{h}^{4}}\right) .
\end{aligned}
$$

(the actual values of $p_{1}$ and $r_{ \pm}$will not be needed). As for $F_{m}^{p}$, the corresponding formulas are rather cumbersome for an arbitrary $0 \leq k_{h}<1$. Fortunately, in this Section we will need primarily their asymptotics at $\lambda^{2} \rightarrow 1$, cf. App. A

$$
\begin{aligned}
& \frac{1}{3} F_{1}^{p_{1}}(\lambda) \simeq \frac{1}{\sqrt{1-k_{h}^{2}}}+D_{1} \sqrt{\lambda^{2}-1}, \quad D_{1}=-\frac{E\left(k_{h}\right)}{1-k_{h}^{2}}, \\
& \frac{1}{7} F_{3}^{r_{ \pm}}(\lambda) \simeq \frac{\left(1-C_{ \pm}\right)^{-1}}{\sqrt{1-k_{h}^{2}}}+D_{ \pm} \sqrt{\lambda^{2}-1}, \quad D_{ \pm}=\frac{\left(1-k_{h}^{2}+2 C_{ \pm}^{2}-2 C_{ \pm} k_{h}^{2}\right) E\left(k_{h}\right)-\left(1-C_{ \pm}\right)\left(1-k_{h}^{2}\right) K\left(k_{h}\right)}{2\left(1-C_{ \pm}\right) C_{ \pm}\left(k_{h}^{2}-C_{ \pm}\right)\left(1-k_{h}^{2}\right)},
\end{aligned}
$$

where $K$ and $E$ are again the complete elliptic inte$\operatorname{grals}^{22}$

The series (38) is designed to satisfy the Laplace equation $\left(\partial^{2} / \partial z^{2}+\nabla^{2}\right) \Phi(\mathbf{r}, z)=0$ term by term (cf. Refs. 1 or 27). Therefore, our task is to demonstrate that with a suitable choice of $\alpha_{m}^{p}, \Phi(\mathbf{r}, z)$ also satisfies the boundary conditions generated by Eqs. (11), (2), (36), and (37):

$$
\begin{aligned}
\frac{\partial}{\partial z} \Phi(\mathbf{r}, z= \pm 0) & =\mp 2 \pi(e / \kappa) \sigma(\mathbf{r}), \quad \mathbf{r} \in S, \\
\Phi(\mathbf{r}, z=0) & =0, \quad \mathbf{r} \notin S .
\end{aligned}
$$

In the last line we set $\mu_{e}$ to zero, for convenience.

In order to express these boundary conditions in terms of $\lambda, \mu$, and $\nu$ we note that the points immediately above and below the ellipse $S$ [Eq. (37)] correspond to $\lambda^{2} \rightarrow$ $1+0$. Similarly, points immediately above (below) the rest of the $2 \mathrm{D}$ plane correspond to $\mu^{2} \rightarrow 1-0$. The condition (45) will be satisfied if $\Phi=\Phi_{1}+\Phi_{2}$, where $\Phi_{1}$ is analytic in $\lambda^{2}$ at $\lambda^{2}=1$, while $\Phi_{2}$ has a square-root singularity,

$$
\Phi_{2} \underset{\lambda^{2} \rightarrow 1}{\sim}-\frac{2 \pi e a \sigma}{\kappa \sqrt{1-k_{h}^{2}}} \sqrt{\left(\lambda^{2}-1\right)\left(1-\mu^{2}\right)\left(1-\nu^{2}\right)} .
$$

Expressed as a function of $\mu$ and $\nu, \sigma$ takes the form

$$
\begin{aligned}
\sigma(\mu, \nu) & =\sigma_{0}+\frac{1}{2} \sigma_{y y} a^{2}\left(\mu^{2}+\nu^{2}-k_{h}^{2}\right) \\
& +\frac{1}{2} \frac{a^{2}}{k_{h}^{2}}\left(\sigma_{x x}-\sigma_{y y}\right) \mu^{2} \nu^{2} .
\end{aligned}
$$

Finally, Eq. 46) is equivalent to

$$
\Phi(\lambda, \mu=1, \nu)=0
$$

A quick examination of Eqs. (47) and 48) makes the above claim that the series (38) involves only the ellipsoidal harmonics defined by Eqs. (41)- (44) plausible. Assuming that this is true, we conclude that on the ellipse $S, \Phi$ must factorize as follows:

$$
\Phi=\sqrt{\left(1-\mu^{2}\right)\left(1-\nu^{2}\right)} P\left(\mu^{2}, \nu^{2}\right),
$$

where $P$ is a polynomial of the second degree symmetric in its two arguments. Since $\Phi$ must satisfy Eq. (49), it has to be of the form

$$
\Phi=\frac{\Phi_{0}}{\left(1-k_{h}^{2}\right)^{3 / 2}}\left(1-\mu^{2}\right)^{3 / 2}\left(1-\nu^{2}\right)^{3 / 2}, \quad \lambda=1,
$$

where $\Phi_{0}$ is some constant. Combined with the expressions for $F_{m}^{p}(1)$ that follow from Eqs. (43) and (44), this fixes the expansion coefficients $\alpha_{m}^{p}$ to be

$$
\begin{aligned}
\alpha_{1}^{p_{1}} & =-\frac{1}{5} \Phi_{0}, \\
\alpha_{3}^{r_{-}} & =-\alpha_{3}^{r_{+}}=\frac{3}{14} \frac{\Phi_{0}}{\sqrt{1-k_{h}^{2}+4 k_{h}^{4}}} .
\end{aligned}
$$

After some more algebra one finds that the boundary condition (47) can indeed be satisfied if $k_{h}$ is the solution of the equation

$$
\frac{\sigma_{x x}}{\sigma_{y y}}=\left(1-k_{h}^{2}\right) \frac{\left(2 k_{h}^{2}-1\right) E\left(k_{h}\right)+\left(1-k_{h}^{2}\right) K\left(k_{h}\right)}{\left(1+k_{h}^{2}\right) E\left(k_{h}\right)-\left(1-k_{h}^{2}\right) K\left(k_{h}\right)},
$$

while $a$ is set by

$$
a^{2}=-\frac{2 \sigma_{0}}{\sigma_{y y}} \frac{\left(1+k_{h}^{2}\right) E\left(k_{h}\right)-\left(1-k_{h}^{2}\right) K\left(k_{h}\right)}{k_{h}^{2}\left(1-k_{h}^{2}\right) E\left(k_{h}\right)} .
$$


For the potential $\Phi_{0}$ at the center of the DR we get

$$
\Phi_{0}=\frac{2 \pi}{3} \frac{e}{\kappa} \frac{k_{h}^{2}\left(1-k_{h}^{2}\right)^{3 / 2}}{\left(1+k_{h}^{2}\right) E-\left(1-k_{h}^{2}\right) K} \sigma_{y y} a^{3},
$$

while at other points on $S, \Phi(\mathbf{r})$ takes the form stipulated by Eq. (51):

$$
\Phi(\mathbf{r})=\Phi_{0}\left(1-\frac{x^{2}}{a^{2}}-\frac{y^{2}}{b^{2}}\right)^{3 / 2} \quad(\mathbf{r} \in S) .
$$

At large distances from the origin, $\Phi(\mathbf{r}, z)$ is dominated by the $m=1, p=p_{1}$ term in Eq. (38) and behaves similar to the potential of an electric dipole,

$$
\Phi(\mathbf{r}, z) \sim \frac{p_{h}}{\kappa} \frac{|z|}{\left(r^{2}+z^{2}\right)^{3 / 2}}, \quad p_{h}=\frac{4 \pi}{15} \frac{e}{E} \sigma_{0} a b^{2},
$$

except the apparent dipole moment $\pm p_{h} \hat{\mathbf{z}}$ has the opposite direction at observation points above and below the $z=0$ plane. Our formula for $p_{h}$ resembles a well known result $\frac{18.19}{19}$ for the apparent dipole moment of a round hole in a metallic sheet.

The sought density distribution $n(\mathbf{r})$ outside of $S$ is given by

$$
n(\mathbf{r})=\sigma(\mathbf{r})+\frac{\kappa}{2 \pi e} \lim _{z \rightarrow 0} \frac{\Phi}{|z|},
$$

or, equivalentely, by

$$
n=\sigma+\frac{\kappa}{2 \pi e a} \lim _{\mu^{2} \rightarrow 1} \frac{\Phi(\lambda, \mu, \nu) \sqrt{1-k_{h}^{2}}}{\sqrt{\left(\lambda^{2}-1\right)\left(1-\mu^{2}\right)\left(1-\nu^{2}\right)}} .
$$

At large $r$ we can use Eq. (58) to obtain

$$
n(\mathbf{r}) \simeq \sigma(\mathbf{r})+\frac{p_{h}}{2 \pi e} \frac{1}{r^{3}}, \quad r \gg a,
$$

which elucidates how the approach to the perfect screening, $n(\mathbf{r}) \rightarrow \sigma(\mathbf{r})$, takes place away from the DR. As for $n(\mathbf{r})$ near the $\mathrm{DR}$, it is rather complicated, except in two cases: (1) a round depletion hole and (2) a slit infinite in the $x$-direction. In the first case the solution is given by Eq. (12) (see App. \$ for details); in the second case, realized at $\sigma_{x x}=0$, the solution is given by Eq. (14) upon replacements $x \rightarrow y, a \rightarrow b$.

It is also possible to work out analytically the lowestorder corrections to these two limiting cases. Thus, a highly asymmetric $\sigma$-minimum, $z_{h} \equiv \sigma_{x x} / \sigma_{y y} \ll 1$, gives rise to a strongly elongated $\mathrm{DR}$ with parameters

$$
\begin{aligned}
a^{2} & =-\frac{2 \sigma_{0}}{\sigma_{x x}}\left[1+\mathcal{L} z_{h}+o\left(z_{h}\right)\right], \quad \mathcal{L} \equiv \ln z_{h}^{-1}-1, \\
b^{2} & =-\frac{4 \sigma_{0}}{\sigma_{y y}}\left[1-\frac{1}{2} \mathcal{L} z_{h}+o\left(z_{h}\right)\right] \\
\Phi_{0} & =-\frac{8 \pi}{3} \frac{e}{\kappa} \frac{\left|\sigma_{0}\right|^{3 / 2}}{\sigma_{y y}^{1 / 2}}\left[1-\frac{1}{2} \mathcal{L} z_{h}+o\left(z_{h}\right)\right] .
\end{aligned}
$$

Equation (15) is recovered in the limit $z_{h} \rightarrow 0$.
On the other hand, if the $\sigma$-minimum is nearly axially symmetric, $\delta z \equiv 1-z_{h} \ll 1$, then the corresponding DR is nearly circular, with the parameters

$$
\begin{aligned}
a^{2} & =-\frac{3 \sigma_{0}}{\sqrt{\sigma_{x x} \sigma_{y y}}}\left[1+\frac{2}{5} \delta z+O\left(\delta z^{2}\right)\right], \\
b^{2} & =-\frac{3 \sigma_{0}}{\sqrt{\sigma_{x x} \sigma_{y y}}}\left[1-\frac{2}{5} \delta z+O\left(\delta z^{2}\right)\right], \\
\Phi_{0} & =-\frac{8}{\sqrt{3}} \frac{e}{\kappa} \frac{\left|\sigma_{0}\right|^{3 / 2}}{\left(\sigma_{x x} \sigma_{y y}\right)^{1 / 4}}+O\left(\delta z^{2}\right) .
\end{aligned}
$$

At $\delta z=0$, Eqs. (65) and (66) reduce to Eq. (111).

Let us now discuss the effect of an isolated elliptic DR on the total energy of the system. It is easy to see that the DR causes a positive correction $\Delta E$ to the energy, which can be estimated as follows. The unscreened electric field that surrounds the DR is of the order of $\mathcal{E} \sim 2 \pi(e / \kappa) \sigma_{0}$ and is concentrated mainly in a volume $\Delta V \sim a \times b \times b$. Hence,

$$
\Delta E=\frac{\kappa}{8 \pi} \int d^{2} r \int d z \mathcal{E}^{2}(\mathbf{r}, z) \sim \frac{e^{2}}{\kappa} \sigma_{0}^{2} a b^{2} .
$$

The exact calculation of $\Delta E$ is more convenient to perform in terms of the potential $\Phi(\mathbf{r})$ and the total charge density $e n(\mathbf{r})-e \sigma(\mathbf{r})$,

$$
\begin{aligned}
\Delta E & =\frac{1}{2} \int d^{2} r \Phi(\mathbf{r}) e[n(\mathbf{r})-\sigma(\mathbf{r})] \\
& =\frac{1}{2} e \Phi_{0} \int_{S} d^{2} r \sigma(\mathbf{r})\left(1-\frac{x^{2}}{a^{2}}-\frac{y^{2}}{b^{2}}\right)^{3 / 2} \\
& =\frac{16 \pi^{2}}{105} \frac{e^{2}}{\kappa} \frac{\sigma_{0}^{2} a b^{2}}{E\left(k_{h}\right)} .
\end{aligned}
$$

The result is in agreement with the preceding estimate. [Recall that $1 \leq E\left(k_{h}\right) \leq \pi / 2$, and so $E\left(k_{h}\right)$ does not have a strong dependence on $a / b$.] For fixed $\sigma_{x x}$ and $\sigma_{y y}$, we have $a, b \propto\left|\sigma_{0}\right|^{1 / 2}$, so that $\Delta E \propto\left|\sigma_{0}\right|^{7 / 2}$.

The energy correction due to DRs affects a number of experimentally measurable properties of $2 \mathrm{D}$ electron systems. One example is the magnetization of quantum dots and quantum wires under the quantum Hall effect conditions. Although the DRs with $n(\mathbf{r})=0$ do not appear in that context, a very similar role is played by regions of locally depleted topmost Landau level 3.9 .

Another example concerns the DRs induced by random impurities, see Fig. 1] Such DRs can strongly influence the energy density of macroscopic 2D systems, especially at low electron densities. This effect may be important 11 for the actively studied phenomenon of the $2 \mathrm{D}$ metalinsulator transition 28

To show how the obtained formulas can be applied in this context let us discuss one experimental technique, which is a particular sensitive probe of DRs. This technique, pioneered by Eisenstein $\frac{29}{,}$ is the measurement of the electric field penetration. In order to do this type of experiment, $\stackrel{29.30 .31}{ }$ one prepares a structure where the 


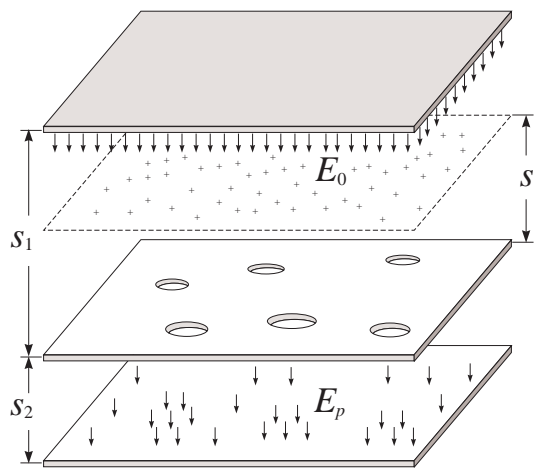

FIG. 1: The geometry of the field-penetration experiment. The 2D layer of interest is sandwiched between the top and the bottom metallic gates. The sample contains ionized impurities (dopants) that are shown as randomly scattered plus signs. The case of $\delta$-doping is assumed where all the dopants reside in a plane parallel to the $2 \mathrm{D}$ layer. The impurities create a random electric field that nucleates the depletion regions (shown as holes in the probed layer). These depletion regions enhance the penetrating electric field $E_{p}$ that can be detected with the help of the bottom gate.

2D layer is sandwiched between two electrodes that can be considered good metals. Once a voltage difference is applied between the 2D layer and the top electrode, some electric field leaks through the 2D layer and its spatial average $\left\langle E_{p}\right\rangle$ can be measured by monitoring the amount of electric charge that has flown into the bottom layer, see Fig. I It is immediately obvious that DRs must enhance $\left\langle E_{p}\right\rangle$. Using the results of this Section, we are now able to calculate how this enhancement of $\left\langle E_{p}\right\rangle$ is related to the concentration and the linear sizes of the DRs. In this calculation we will assume that the interlayer distances $s_{1}$ and $s_{2}$ (see Fig. 1) are large.

Equation (58) gives one way to calculate $\left\langle E_{p}\right\rangle$. An alternative and a more general way is to work with the energy correction $\Delta E$ and the corresponding contribution $\Delta C^{-1}$ to the inverse capacitance per unit area of the device. $\left[\Delta C^{-1}\right.$ is the correction to the inverse geometric capacitance $C_{0}^{-1}=4 \pi s_{1} / \kappa$, cf. Eq. [6])]. For a single elliptic DR $\Delta E$ and $\Delta C^{-1}$ are related by

$$
\Delta C^{-1}=\frac{A}{e^{2}} \frac{d^{2} \Delta E}{d N_{e}^{2}}=\frac{1}{e^{2} A} \frac{d^{2} \Delta E}{d \sigma_{0}^{2}}=\frac{4 \pi^{2}}{3 \kappa A} \frac{a b^{2}}{E\left(k_{h}\right)},
$$

where $A$ is the sample area. If the DRs in the 2D layer are well separated, their contributions to $\Delta C^{-1}$ are, in the first approximation, additive. The total correction to the inverse capacitance, $\Delta C_{t o t}^{-1}$, characterizes the nonideal nature of our capacitor and therefore the amount of field penetration. Indeed, a simple derivation along the lines of Ref. 29 leads to the relation

$$
\frac{d}{d n_{e}}\left\langle E_{p}\right\rangle=\frac{e}{s_{2}} \Delta C_{t o t}^{-1}=\frac{4 \pi^{2}}{3} \frac{e}{\kappa} \frac{N_{\mathrm{DR}}}{s_{2}}\left\langle\frac{a b^{2}}{E\left(k_{h}\right)}\right\rangle,
$$

where $N_{\mathrm{DR}}$ is the DR concentration.
Note that as all the other results in this paper, Eq. (71) is derived in the approximation that ignores non-Hartree terms in the energy (kinetic and exchange-correlation energies). This is not a serious omission at low electron densities $n_{e}$ where the DRs dominate the field penetration. However, at higher densities, the non-Hartree terms must be included. It may lead to an interesting nonmonotonic behavior of $\Delta C_{\text {tot }}^{-1}\left(n_{e}\right)$, discussed theoretically in Refs. 16 and 11 .

\section{SADDLE-POINT}

In this Section we examine the structure of the $2 \mathrm{D}$ electron liquid near a saddle-point of the external potential,

$$
\Phi_{e x t}=\frac{1}{2} U_{y y} y^{2}-\frac{1}{2} U_{x x} x^{2}+O\left(r^{4}\right), \quad U_{x x}, U_{y y}>0 .
$$

This is the most interesting fundamental geometry but also the most difficult one for the analytical study.

It is easy to understand that the electron liquid should be confined in the $y$-direction into a strip that has the smallest width at $x=0$ and widens up as $|x|$ increases. In other words, this structure can be thought of as a local constriction in a $2 \mathrm{D}$ electron system. Such structures have been fabricated and intensively studied in the past. Especially much attention has been devoted to quantum point contacts, which are constrictions with the bottleneck width comparable to the interelectron separation ${ }^{33}$ Nevertheless, to the best of out knowledge, the analytic solution for the electron density profile $n(x, y)$ and the total electrostatic potential $\Phi(x, y)$ around the constriction has not been presented. (For a recent numerical work, see Ref. 34). Instead, the unknown functional forms of $n$ and $\Phi$ have been approximated by expressions chosen somewhat arbitrarily. For $\Phi(\mathbf{r})$, a parabolic function ${ }^{35.36}$ [as in Eq. (72)] or a parabola with a flat insert ${ }^{37}$ have been used. The boundary of the 2D electron channel was modelled variously by combination of wedges,, 38 circular arcs $\stackrel{40}{,}$ confocal hyperbolas, or more complicated curves $\stackrel{41}{\underline{41}}$

Let $y= \pm b(x)$ be the equation for the boundaries of the electron liquid in the constriction. Although we have not succeeded in solving the electrostatics problem for the saddle-point completely, below we show that the correct asymptotical behavior of $b(x)$ is given by

$$
b(x)=\alpha_{0}|x| \exp \left(-\sqrt{\ln \left|\frac{x_{0}}{x}\right|}\right), \quad|x| \ll x_{0},
$$

where $\alpha_{0}, x_{0}$ are constants determined by the boundary conditions far from the saddle-point. This is perhaps the most interesting theoretical result that we achieve in this work.

The exponential factor in Eq. (73) is a nontrivial effect of the long-range Coulomb interaction (see below). It is therefore interesting to do a quick estimate of this factor 
for a typical experimental setup. As explained in Sec. 【 pure electrostatics is not adequate on distances shorter than the interparticle separation $a_{\text {e-e. }}$. Therefore, in reality, Eq. (73) applies only as long as $b(x),|x| \gg a_{\mathrm{e}-\mathrm{e}} \sim$ $1 / \sqrt{n(x, 0)}$. On the other hand, $x_{0}$ can be large. For example, using $x_{0} \sim 1 \mu \mathrm{m}$ and $x=a_{\mathrm{e}-\mathrm{e}} \sim 50 \mathrm{~nm}$, which are reasonable numbers for GaAs-based point contacts, we obtain $\ln \left(x_{0} / x\right) \sim 3.0$, so that the exponential factor in Eq. (73) is approximately 0.2. Thus, the long-range interaction can cause a significant narrowing of the bottleneck of the constriction.

In the purely electrostatic model $\left(a_{\mathrm{e}-\mathrm{e}}=0\right)$ we are allowed to consider the limit $x \rightarrow 0$. Then Eq. (73) predicts that the tangent $\alpha(x)=b(x) / x$ of the opening angle of the constriction becomes vanishingly small, independently of $U_{y y} / U_{x x}$. This behavior stems from the long-range nature of the Coulomb interaction between the electrons. With short-range interactions (or without interactions) electrons fill the constriction up to the equipotential contour $\Phi_{e x t}(x, y)=\mu_{e}$; therefore, the constriction is either bounded by two confocal hyperbolas or, when $b(0) \rightarrow 0$, by the two straight lines. In the latter case $\alpha=\left(U_{x x} / U_{y y}\right)^{1 / 2}$, which is finite, in contrast to Eq. (73). The physical reason for the difference is the ability of the system with long-range interactions to modify (screen) the external potential even at points $\mathbf{r}=(x, y)$ that are outside of the area occupied by the electrons. The screening flattens out the confining potential $\Phi_{\text {ext }}$, allowing the electron liquid to spread over a larger area compared to the noninteracting limit. The nontrivial statement embodied by Eq. (73) is that the screening is more effective along the longitudinal $(x)$ direction, so that the extra area occupied by the interacting electrons is strongly funneled into the constriction center.

Let us proceed to the derivation of Eq. (73). We distinguish two cases, which are treated in two separate subsections below.

\section{A. Small opening-angle constriction}

First we will treat the limit $U_{x x} \ll U_{y y}$ where $\Phi_{e x t}$ is a slow function of $x$. In this case the constriction can be thought of as an adiabatically narrowing quantum wire $\stackrel{42}{2}$ To the lowest order in parameter $U_{x x} / U_{y y}$, the $y$-dependence of $n(x, y)$ at a given fixed $x$ can be found by solving the requisite electrostatic problem assuming that $\Phi_{\text {ext }}$ is $x$-independent. The solution for $n(\mathbf{r})$ is given by Eq. (32) where $b$ should now be understood as a yet to be found function of $x$. The electrostatic potential is also easy to find,

$$
\Phi(\mathbf{r})=\mu_{e}+\frac{1}{2} U_{y y}\left(y \sqrt{y^{2}-b^{2}}-b^{2} \cosh ^{-1} \frac{y}{b}\right) .
$$

Let us define the one-dimensional (1D) electron density $q(x)$ along the wire by

$$
q(x)=\int_{-b}^{b} d y n(x, y)=\frac{1}{4} \frac{\kappa}{e^{2}} U_{y y} x^{2} \alpha^{2}(x) .
$$

In view of the last equation, the desired $b(x)$ is directly related to $q(x)$. To find the equation for $q(x)$ we substitute Eq. (32) into the boundary condition (II) and take $y=0$. This yields

$$
\int_{-x_{0}}^{x_{0}} d x^{\prime} G\left(x, x^{\prime}\right) q\left(x^{\prime}\right)=\frac{1}{2} U_{x x} x^{2}-\mu_{e}-\Delta \Phi\left(x_{0}\right) .
$$

The kernel $G\left(x, x^{\prime}\right)$ can be expressed in terms of elliptic integrals. At $\left|x-x^{\prime}\right| \gg b\left(x^{\prime}\right)$, it reduces to the Coulomb interaction potential $G\left(x, x^{\prime}\right) \simeq e^{2} / \kappa\left|x-x^{\prime}\right|$. The term $\Delta \Phi\left(x_{0}\right)$ in Eq. (76) represents the potential created by electrons located at points $|x|>x_{0}$. It is determined by the behavior of $\Phi_{\text {ext }}$ at large distances and therefore high energies. On the other hand, we are interested mainly in the structure of the constriction as small $x$. Physically, we may expect that minor changes in $\mu_{e}$ modify $b(x)$ near the origin considerably but leave $\Delta \Phi\left(x_{0}\right)$ virtually the same. Therefore, the role of $\Delta \Phi\left(x_{0}\right)$ is simply to renormalize the electrochemical potential by a constant: $\mu_{e} \rightarrow \mu_{e}+\Delta \Phi\left(x_{0}\right)$.

The idea of renormalization proves to be very fruitful in the present problem. Indeed, since we are interested in the behavior at small distances, we have a freedom in choosing the cutoff as long as it exceeds the distance of interest. Then $\mu_{e}$ should be viewed as a function of the cutoff and as such, it must satisfy a certain renormalization group (RG) equation. Similarly, there must be an RG equation for $U_{x x}$ and, in fact, for $U_{y y}$. From Eq. (76) one finds that to the lowest order in the parameter $\alpha(l) \ll 1$, these equations are

$$
\frac{d}{d l} U_{x x}=-U_{y y} \alpha^{2}(l), \quad \frac{d}{d l} U_{y y}=-\frac{1}{2} U_{y y} \alpha^{2}(l),
$$

where $l=\ln \left|x_{0} / x\right|, x$ being the running cutoff. The RG flow persists as long as $\frac{43}{3}|x| \gg b(x)$. Let us consider the most interesting case, $b(0)=0$, where $|x|$ is always larger than $b$, so that the RG is able to reach its fixed point. What is this fixed point? To the order we are working with, $U_{y y}=U_{y y}\left(x_{0}\right)+\left[U_{x x}-U_{x x}\left(x_{0}\right)\right] / 2$. This implies that for $U_{y y}\left(x_{0}\right) \gg U_{x x}\left(x_{0}\right)$ the renormalization of $U_{y y}$ can be ignored. Therefore, at the fixed point, where the right-hand sides of Eq. (77) must vanish, $\alpha(l=\infty)=$ $\alpha(x=0)=0$, in agreement with Eq. (73) and statements above.

Let us now derive the complete form of $\alpha(x)$. From Eqs. (75) and (76) we find that

$$
-\alpha^{2}(l)[\ln \alpha(l)+O(1)]+\int_{0}^{l} d l^{\prime} \alpha^{2}\left(l^{\prime}\right)=\frac{U_{x x}\left(x_{0}\right)}{U_{y y}} .
$$


Differentiating both sides with respect to $l$, we get

$$
\frac{d}{d l} \alpha \simeq \frac{\alpha}{2 \ln \alpha},
$$

which complements Eq. (77) and has (73) as the solution.

It is instructive to rewrite Eq. (73) in terms of the renormalized $U_{x x}$ [this can be done by integrating Eq. (77)]:

$$
\alpha^{2}(l)=2 \frac{U_{x x}(l)}{U_{y y}}\left[1+\ln \frac{U_{x x}(0)}{U_{x x}(l)}\right]^{-1} .
$$

Since $U_{x x}(l=\infty)=0$ [see Eqs. (77) and (78)], Eq. (80) describes the decrease of $\alpha(x)$ from its noninteracting limit value at $x=x_{0}$ to the asymptotical zero at $x=0$.

The obtained RG fixed point must have a finite basin of attraction, presumably $\alpha \lesssim 1$, for which Eq. (73) must be valid. The strong evidence that Eq. (73) is the universal asymptotical law independently of the starting $\alpha\left(x_{0}\right)$ is furnished by the following analysis of the case $\alpha\left(x_{0}\right) \gg 1$, which is the far departure from the found fixed point.

\section{B. Large opening-angle constriction}

If the starting, i.e., long-distance value of $\alpha$ is large, it is no longer convenient to parametrize the saddle-point by $U_{x x}$ and $U_{y y}$. Indeed, now the external potential $\Phi_{e x t}$ is almost completely screened, and so it is rather useless in setting up the problem. Instead, as in Sec. IIII we describe the effect of the external sources by an effective neutralizing charge density $\sigma$, so that the total charge density in the system is $n(\mathbf{r})-\sigma(\mathbf{r})$, see Sec. [1. Near the origin, $\sigma(\mathbf{r})$ should be of a saddle-point type,

$$
\sigma=\sigma_{0}+\frac{1}{2} \sigma_{x x} x^{2}-\frac{1}{2} \sigma_{y y} y^{2}, \quad 0<\sigma_{y y} \ll \sigma_{x x} .
$$

In fact, working with $\sigma(\mathbf{r})$ instead of $U(\mathbf{r})$ also brings us closer to the practical side of fabrication of such a largeangle constriction. It seems feasible that one can make this type of a structure by depositing a static surface charge of the form (81) nearby the 2D plane but doing it with voltage sources (thin metallic gates) may be problematic. Although in the former method $\sigma_{x x}$ and $\sigma_{y y}$ in Eq. (81) would likely be fixed once the structure is made, $\sigma_{0}$ could still be varied by an additional distant gate on top of the device.

As in the case of the small-angle constriction, the relation between $\sigma_{0}$ and $\mu_{e}$, i.e., the top-gate voltage in the suggested setup, is determined by behavior at large distances. Consequently, in a small interval of $\mu_{e}$ of interest to us we should have

$$
\sigma_{0}=\left(C / e^{2}\right) \mu_{e},
$$

where $C$ is a constant approximately equal to $C_{0}$, the geometric capacitance per unit area [cf. Eq. [6)].
We have a situation where the $2 \mathrm{D}$ plane is almost completely covered by the metallic liquid, except a narrow depletion strip that gradually fans out of the origin along the $y$-axis. This problem is adiabatic with respect to coordinate $y$. To the lowest order in $1 / \alpha_{1}$, where

$$
\alpha_{1}=\left(\sigma_{x x} / 2 \sigma_{y y}\right)^{1 / 2},
$$

we can solve the system of Eqs. (11), (21), (4), and (81) pretending that $\sigma$ is $y$-independent. The solution is an obvious modification of Eqs. (14) and (57):

$$
\begin{aligned}
n(x, y) & =\frac{1}{2} \sigma_{x x}|x| \sqrt{x^{2}-a^{2}(y)}, \\
a^{2}(y) & =-4 \sigma(0, y) / \sigma_{x x}, \\
\Phi(x, y) & =\mu_{e}+\frac{\pi}{3} \frac{e^{2}}{\kappa} \sigma_{x x}\left[a^{2}(y)-x^{2}\right]^{3 / 2} .
\end{aligned}
$$

These equations apply whenever they give real $n$ and $\Phi$; otherwise, $n=0$ and $\Phi=\mu_{e}$. At this level of aproximation, the boundaries of the constriction are confocal hyperbolas defined by the equation $x^{2}=a^{2}(y)$, see Eq. 855. At $\sigma_{0}=0$ where the constriction just opens up, these hyperbolas become straight lines with $\alpha=\alpha_{1}=$ const, while $\Phi$ acquires the cubic dependence on $y$ :

$$
\Phi(0, y)=\frac{\pi}{24} \frac{e^{2}}{\kappa} \sigma_{y y}^{3 / 2} \sigma_{x x}^{-1 / 2}|y|^{3} .
$$

Let us now show that in a more careful treatment, Eqs. 844- 87) become invalid at exponentially small $x$ and $y$. It is convenient to assume that at large $y, \sigma(\mathbf{r})$ changes from the decreasing to an increasing function of $y$ so that the depletion region terminates at some $|y|=y_{0}$. In this case the system can be thought of as a $2 \mathrm{D}$ metallic sheet with an elongated bowtie-shaped hole. Similar to the case of a round hole,$\stackrel{44}{=}$ the $3 \mathrm{D}$ electrostatic potential at $|x|,|z| \gg a(y)$ can be sought in the form

$$
\Phi(\mathbf{r}, z)-\mu_{e} \simeq \frac{e^{2}}{\kappa} \int_{-y_{0}}^{y_{0}} \frac{d y^{\prime} p\left(y^{\prime}\right)|z|}{\left[x^{2}+z^{2}+\left(y-y^{\prime}\right)^{2}\right]^{3 / 2}},
$$

which is the lowest-order (dipolar) term in the multipole expansion compatible with Eq. (11). Since the total charge density $\Delta n(\mathbf{r})=n(\mathbf{r})-\sigma(\mathbf{r})$ is proportional to the discontinuity in $\partial \Phi / \partial z$ at $z=0$, Eq. (88) entails

$$
\Delta n(\mathbf{r}) \simeq \frac{1}{2 \pi} \int_{-y_{0}}^{y_{0}} \frac{d y^{\prime} p\left(y^{\prime}\right)}{\left[x^{2}+\left(y-y^{\prime}\right)^{2}\right]^{3 / 2}} .
$$

To find $p(y)$ we match the near and far-field asymptotics, Eqs. (14) and (89). To the lowest order in $1 / \alpha_{1}$, we get

$$
p(y)=-(\pi / 16) \sigma_{x x} a^{4}(y) .
$$

Next, as in the case of a narrow constriction, we must account for the renormalization $\sigma \rightarrow \sigma+\Delta \sigma$ of the bare 
parameters. From Eqs. (89) and (90) we obtain

$$
\Delta \sigma(0, y)=\frac{\sigma_{x x}}{32} \int_{-y_{0}}^{y_{0}} \frac{d y^{\prime}\left[a^{4}\left(y^{\prime}\right)-a^{4}(y)\right]}{\left|y-y^{\prime}\right|^{3}+c a^{3}(y)}, \quad c \sim 1 .
$$

Evaluating this integral and substituting $\Delta \sigma(0, y)$ into Eq. 85., we find a divergent log-correction to $\alpha=y / a$. To facilitate the comparison with Eq. (73), the final result can be presented in the form

$$
b(x)=\alpha_{1}|x|\left(1-\frac{12}{\alpha_{1}^{2}} \ln \left|\frac{x_{0}}{x}\right|\right) .
$$

The most likely behavior consistent with both Eqs. (73) and (92) is as follows. A constriction that appears very wide, $\alpha=\alpha_{1} \gg 1$, at large distances, renormalizes first into an $\alpha \sim 1$ structure at $x=x_{c}$,

$$
x_{c} \sim x_{0} \exp \left(-\alpha_{1}^{2} / 12\right),
$$

and then into an adiabatically narrowing small openingangle constriction at even smaller $x$. If so, Eq. (73) is a universal asymptotic law.

Concomitantly, we expect that Eq. (94) applies only at $|y| \gg x_{c}$; otherwise, it is replaced by

$$
\Phi(0, y)=\frac{e^{2}}{\kappa} \frac{\sigma_{y y}^{3 / 2}}{\sigma_{x x}^{1 / 2}} x_{c} y^{2}, \quad|y| \ll x_{c},
$$

in a nominal agreement with the popular models ${ }^{35.36}$ for the electrostatic potential near the constriction.

\section{Numerical results}

In order to verify the outlined analytical theory we done a series of numerical simulations. In these simulations the sought $n(x, y)$, the external potential in the form $\Phi_{e x t}=-(1 / 2) U_{x x} x^{2}+(1 / 2) U_{y y} y^{2}+U x^{4}$, and the Coulomb interaction kernel were discretized on a realspace square grid. The units used in the simulations were $e=\kappa=1$, and the length unit was the size of the grid cell. In each run, the energy of the system was minimized numerically for a trial $\mu_{e}$ with the help of MAT$\mathrm{LAB}^{\mathrm{TM}}$ QUADPROG library function. The value of $\mu_{e}$ at which the constriction just opens up was then found by minimizing the combination $\left(\Phi-\mu_{e}\right)^{2}+n^{2}$ at the point $\mathbf{r}=(0,0)$. To avoid a difficult task of reconstructing $b(x)$ from $n$-data defined on the discrete grid, $n(x, 0)$ was studied instead. According to Eq. (32), $n(x, 0) \propto b(x)$, so that the scaling form Eq. (73) should equally apply to $n(x, 0)$. Shown in Fig. 2 2 a) is the numerically calculated $n(x, 0)$ for the $U_{x x}=1, U_{y y}=4$, the grid size $161 \times 81$, and the system size $|x| \leq 1 / 2,|y| \leq 1 / 4$. On the same graph we plot the best fit to Eqs. (73) and (32), which is obtained for $\frac{45}{\underline{4}} \alpha_{0}=1.15, x_{0}=0.82$. Considering that for the chosen simulation parameters we are not yet deep inside the asymptotic regime $\alpha \ll 1$ [in the interval of
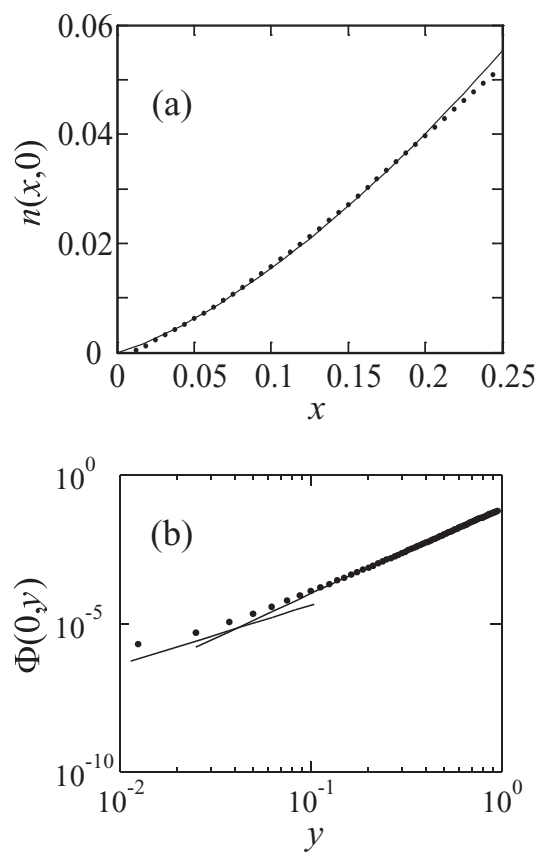

FIG. 2: (a) Numerical (dots) and analytical (solid line) results for $n(x, 0)$ at $U_{x x}=1, U_{y y}=4, U=2$. (b) $\Phi(0, y)$ (dots) at $U_{x x}=1, U_{y y}=0.2, U=3.125$. The slopes for quadratic and cubic dependences are shown for comparison.

$x$ shown in Fig. 2(a), $\alpha$ calculated according to Eq. (32) ranges from 0.5 to about 0.2 , the quality of the fit is quite acceptable. In other words, we think that our computer simulations do support the validity of Eq. (73).

In Fig. 2(b), $\Phi(0, y)$ for the case $U_{x x} / U_{y y}=5$ is presented. In agreement with Eq. 877, it shows a cubic $y$-dependence at large $y$ and is more consistent with the quadratic law of Eq. (94) at small $y$. This again reinforces our case for universality of Eq. (733).

Concluding this Section, we would like to make a few brief comments on the relevance of the obtained results for experiments. First, our predictions for $n(\mathbf{r})$ and $\Phi(\mathbf{r})$ can be directly verified by a number of currently available high-resolution imaging techniques, e.g., near-field

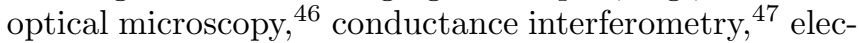
trostatic force microscopy, ${ }_{48}^{48}$ or local potentiometry ${ }^{31.32}$ Second, it is feasible that the differences between the found solution and commonly assumed forms of $n$ and $\Phi$ can also be detected in transport through a quantum point contact. The signatures of such deviations and the question of how they could be amplified by a suitable design of the point contact warrant further study.

\section{CHECKERBOARD}

As an application of the obtained results to a more complicated geometry, in this Section we examine the $2 \mathrm{D}$ electron liquid situated on the periodic charge-density 
(a)

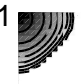

$>0.5$

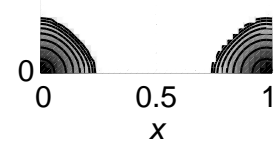

(c)

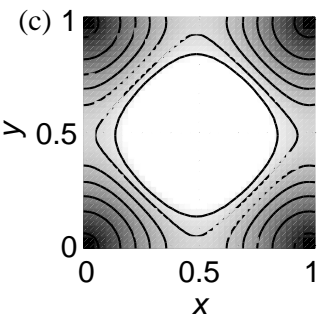

(b)

$>0.5$
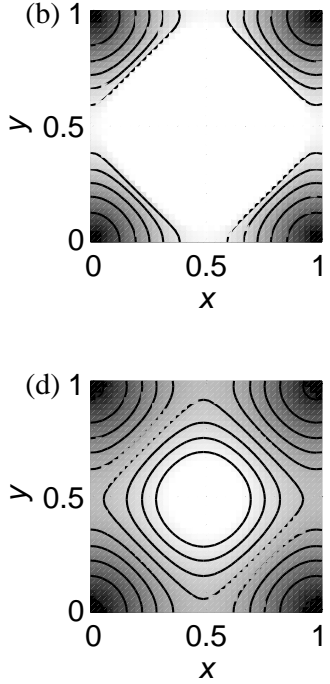

FIG. 3: The ground-state density distribution computed numerically on a $40 \times 40$ square grid for the symmetric checkerboard $b_{x}=b_{y}=1$. Darker areas correspond to higher $n(\mathbf{r})$. The area shown in each subplot extends beyond the boundary of the unit cell by a half grid cell in each direction. (a) low density, $n_{e}=0.0873 n_{1}$. (b) $n_{e}=0.246 n_{1}$, which is slightly above $n_{p}$. (c) $n_{e}=0.470 n_{1}$, about twice larger than $n_{p}$ (d) high density, $n_{e}=0.836 n_{1}$. The solid lines are $n=$ const contours for a set of linearly spaced densities. These densities are different in each subplot and are chosen to minimize uncertainties in the contour positions that arise due to the discreteness of the grid.

background

$$
\sigma=n_{e}+\frac{n_{1}}{2}\left(\cos \frac{2 \pi x}{b_{x}}+\cos \frac{2 \pi y}{b_{y}}\right), \quad b_{x} \geq b_{y} .
$$

We refer to this model as the "checkerboard." It is interesting because it allows one to study the interplay of the three basic building blocks (a droplet, an isolated DR, and a saddle-point) that exist for a general $\sigma(\mathbf{r})$. The complete analytical solution of the electrostatic problem for the checkerboard geometry remains however unknown $\stackrel{49}{*}$ Instead, we will present a numerical solution and will discuss how the results of Secs. [IIIV] can be used to understand its structure. We will also derive some exact asymptotics and finally, at the end of this Section, we will discuss a semianalytic ansatz that reproduces many properties of the numerical solution with a high accuracy, in particular, its energy as a function of $n_{e}$.

We start with numerical results, which are shown in Fig. 3] These plots represent the distribution of $n(\mathbf{r})$ within the unit cell $0<x \leq b_{x}, 0<y \leq b_{y}$ that are computed by a numerical program similar to that described in Sec. IV In this particular simulation $b_{x}=b_{y}$ so that the unit cell is a square.

As one can see from Fig. 3(a), at low density, $n_{e} \ll n_{1}$, the electron liquid is broken into isolated nearly circular

droplets. The droplets surround the maxima of $\sigma(\mathbf{r})$ that are located at the corners of the unit cell. As $n_{e}$ increases at fixed $n_{1}$, the droplets grow. Their boundaries progressively deviate from the circular form as they become funneled towards the nearest saddle-points of $\sigma(\mathbf{r})$, which are located at the midpoints of the edges of the unit cell. At some density $n_{p}$ (percolation point) the droplets merge. In the symmetric checkerboard simulated on $40 \times 40$ grid, this occurs at the average density of

$$
n_{p}=0.22 n_{1}, \quad b_{x}=b_{y} .
$$

From experiments with different grid sizes, we concluded that the above value should be close to the percolation threshold in the continuum limit but no detailed finitesize scaling was attempted.

Figure 3(b) shows the density profile slightly above $n_{p}$ where the continuous path through the electron liquid already exists. At $n_{p}<n_{e}<n_{1}$ the most noticeable change that takes place as $n_{e}$ continues to increase is the contraction of the depletion hole at the center of the unit cell, see Figs. 3(c) and (d). Finally, at $n_{e} \geq n_{1}$ (not shown) the electron liquid becomes free of the DRs and its profile faithfully repeats the background, $n(\mathbf{r})=\sigma(\mathbf{r})$.

In the asymmetric checkerboard, $b_{x}>b_{y}$, the evolution of the ground state with increasing $n_{e}$ is similar, except that the transition to the global percolation takes place in two steps. First, at some density $n_{p y}$ droplets merge into continuous metallic chains that run parallel to the $y$-axis. Subsequently, at $n_{p}>n_{p y}$, the chains become interconnected. This behavior is illustrated in Fig. 4 where we display the results of our simulations for $b_{x} / b_{y}=2$ on the $30 \times 60$ grid. For this grid size, the two aforementioned thresholds were found to be

$$
n_{p y}=0.17 n_{1}, \quad n_{p}=0.31 n_{1}, \quad \frac{b_{x}}{b_{y}}=2 .
$$

Note that in the asymmetric checkerboard the boundaries of the DRs are elongated along the $x$-direction. In particular, the small droplets at low $n_{e}$ and the small DRs at high $n_{e}$ are elliptic in shape.

Our goal in the rest of this Section is to develop analytical approaches that are able to reproduce the above numerical findings.

\section{A. Exact analytical asymptotics}

The structure of the ground state can be determined asymptotically exactly in the two limits, the low density $\left(n_{e} \ll n_{1}\right)$ and the high density $\left(n_{1}-n_{e} \ll n_{1}\right)$.

We start with the low-density case. Let us split the total charge background $\sigma(\mathbf{r})$ into a part with zero mean, $\sigma(\mathbf{r})-n_{e}$, and a uniform charge density $n_{e}$. The former produces the electrostatic potential

$$
\Phi_{1}(\mathbf{r})=-\frac{e n_{1} b_{x}}{2 \kappa}\left(\cos \frac{2 \pi x}{b_{x}}+\frac{b_{y}}{b_{x}} \cos \frac{2 \pi y}{b_{y}}\right) .
$$


(a)

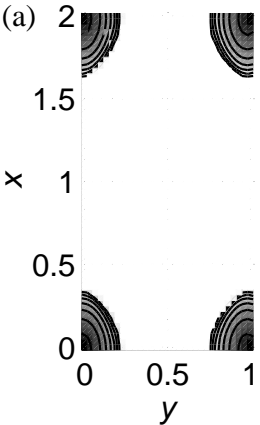

(c)

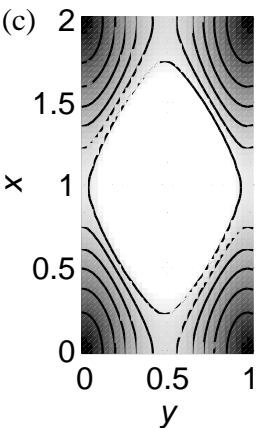

(b)

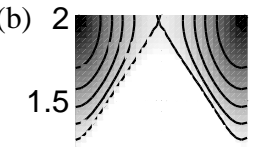

$\times \quad 1$

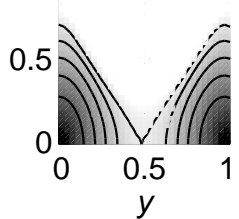

(d)

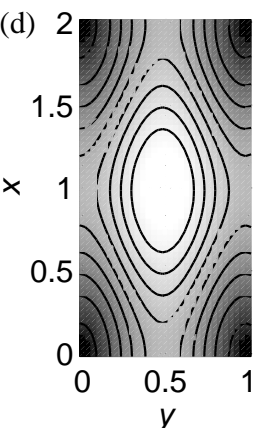

FIG. 4: Similar to Fig. 3 but for $b_{x}=2, b_{y}=1$. (a) low density, $n_{e}=0.0549 n_{1}$. (b) $n_{e}=0.269 n_{1}$, which is between $n_{p y}$ and $n_{p}$. (c) $n_{e}=0.384 n_{1}$, which is above $n_{p}$. (d) high density, $n_{e}=0.897 n_{1}$.

Function $\Phi_{1}(\mathbf{r})$ has the minima at the corners of the unit cell and this is the reason why the metallic droplets that form at small $n_{e}$ reside there. Each droplet has the electric charge $Q=e n_{e} b_{x} b_{y}$. Consider the droplet centered at $(0,0)$ and denote by $\Phi_{\text {ext }}(\mathbf{r})$ the total potential felt by the electrons in that droplet due to all the others and the uniform $n_{e}$ background. To find $\Phi_{\text {ext }}(\mathbf{r})$ we can model the other droplets as point charges $Q$ arranged in the rectangular lattice. In the leading-order approximation in the parameter $n_{e} / n_{1}$, within the small area covered by the droplet, $\Phi_{\text {ext }}$ is related to $\Phi_{1}$ as follows:

$$
\Phi_{e x t}(\mathbf{r})=\Phi_{1}(\mathbf{r})+\frac{Q}{\kappa b_{x}} M\left(\frac{b_{x}}{b_{y}}\right) .
$$

Here $M(z)$ is the Madelung constant of the rectangular lattice with the unit cell of size $1 \times z^{-1}$. $M$ can be easily calculated by the Ewald's method. For example, one finds that $M(1)=-3.900264920001955$. To determine the size of the droplet we further notice that $\Phi_{\text {ext }}$ admits the expansion analogous to that in Eq. (16),

$$
\begin{aligned}
e \Phi_{e x t} & =\mu_{0}+\frac{1}{2} U_{x x} x^{2}+\frac{1}{2} U_{y y} y^{2}+O\left(r^{4}\right), \\
\mu_{0} & =\frac{e^{2} n_{e} b_{y}}{\kappa} M\left(\frac{b_{x}}{b_{y}}\right)-\frac{e^{2} n_{1}}{2 \kappa}\left(b_{x}+b_{y}\right), \\
U_{x x} & =2 \pi^{2} \frac{e^{2} n_{1}}{\kappa b_{x}}, \quad \frac{U_{y y}}{U_{x x}}=\frac{b_{y}}{b_{x}} .
\end{aligned}
$$

Substituting these equations into the formulas of Sec. II we find the semiaxes $a$ and $b$ of the droplet to be

$$
\begin{aligned}
a & =b_{x}\left[\frac{3}{2 \pi^{2}} \frac{K\left(k_{d}\right)-E\left(k_{d}\right)}{k_{d}^{2}} \frac{b_{y}}{b_{x}}\right]^{1 / 3}\left(\frac{n_{e}}{n_{1}}\right)^{1 / 3}, \\
b & =a \sqrt{1-k_{d}^{2}}
\end{aligned}
$$

where $k_{d}$ is the solution of Eq. (22) for $U_{x x} / U_{y y}$ specified by Eq. (102). The depleted area fraction $f_{\mathrm{DR}}$ is related to $a$ and $b$ as follows:

$$
f_{\mathrm{DR}}=1-\frac{\pi a b}{b_{x} b_{y}} .
$$

Using the equation of Sec. III we can also calculate the corrections to the electrochemical potential and the inverse capacitance, $\delta \mu$ and $\Delta C^{-1}$, respectively, in the droplet state:

$$
\begin{aligned}
\Delta C^{-1} & =\left[\frac{2 \pi^{2}}{3} \frac{b_{x} b_{y}^{2} k_{d}^{2}}{K\left(k_{d}\right)-E\left(k_{d}\right)}\right]^{1 / 3}\left(\frac{n_{1}}{n_{e}}\right)^{1 / 3}, \\
\delta \mu & =\frac{3}{2} \frac{e^{2}}{\kappa} \Delta C^{-1} n_{e}+\mu_{0} .
\end{aligned}
$$

Equations (103)- 107) are valid for $\left(b_{x} b_{y}\right)^{-1} \ll n_{e} \ll n_{1}$. At smaller $n_{e}$ one expects deviations due to the discreteness of electrons in each droplet. At larger $n_{e}$ there are other kind of deviations, from the nonelliptic shape of the droplets and their strong mutual interaction.

Let us now switch to the opposite limit of of high density, $\delta n \equiv n_{1}-n_{e} \ll n_{1}$. In this case we deal with small depletion holes that surround the negative minima of $\sigma(\mathbf{r})$. Such minima are located at the centers of the checkerboard cells, e.g., $\left(b_{x} / 2, b_{y} / 2\right)$. Expanding $\sigma(\mathbf{r})$ given by Eq. (95) around this point and adhering to the notations of Eq. (36), we obtain

$$
\begin{aligned}
\sigma_{0} & =n_{e}-n_{1}=-\delta n, \\
\sigma_{x x} & =\frac{2 \pi^{2} n_{1}}{b_{x}^{2}}, \quad \frac{\sigma_{y y}}{\sigma_{x x}}=\frac{b_{x}^{2}}{b_{y}^{2}} .
\end{aligned}
$$

Substituting these expressions into the formulas of Sec. IIII we get the semiaxes of the depletion holes $a$ and $b$ to be

$$
\begin{aligned}
& b=\frac{b_{y}}{\pi}\left[\frac{\left(1+k_{h}^{2}\right) E-\left(1-k_{h}^{2}\right) K}{k_{h}^{2} E\left(k_{h}\right)}\right]^{1 / 2}\left(\frac{\delta n}{n_{1}}\right)^{1 / 2}, \\
& a=\frac{a}{\sqrt{1-k_{h}^{2}}},
\end{aligned}
$$

where $k_{h}$ is the solution of Eq. (54) for $\sigma_{x x} / \sigma_{y y}$ specified by Eq. (109). For the DR area fraction we get

$$
f_{\mathrm{DR}}=\pi \frac{a b}{b_{x} b_{y}}
$$




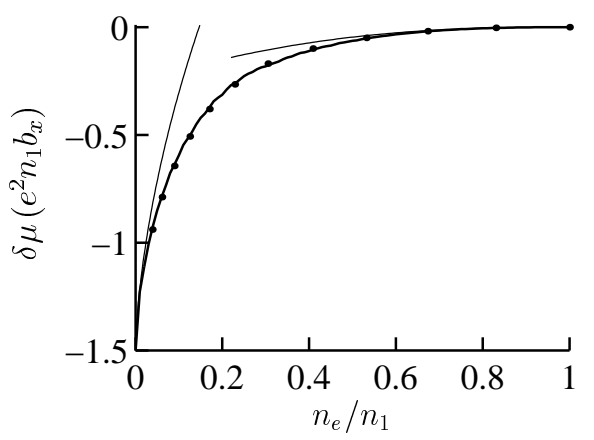

FIG. 5: The electrochemical potential correction $\delta \mu$ according to the analytical asymptotics [Eqs. (107) and (114)] (thin lines), numerical simulations (dots), and the trial ansatz method (thick solid line) for the checkerboard with the unit cell of aspect ratio $b_{x} / b_{y}=2$.

while for $\delta \mu$ and $\Delta C^{-1}$ we find

$$
\begin{aligned}
\Delta C^{-1} & =\frac{4}{3 \pi} \frac{b_{y}^{2}}{b_{x}^{2}} \frac{1-k_{h}^{2}}{E\left(k_{h}\right)}\left(\frac{\delta n}{n_{1}}\right)^{3 / 2}, \\
\delta \mu & =-\frac{2}{5} \frac{e^{2}}{\kappa} \Delta C^{-1} \delta n .
\end{aligned}
$$

So far we neglected the interaction among the depletion holes. In principle, such an interaction, which is a subleading correction of a dipole-dipole type, can be included perturbatively along the lines of Sec. IV However, for all $b_{x} / b_{y}$ studied in our numerical simulations, it was estimated to be a tiny effect at all densities $n_{e}$ at which the approximation of DRs by elliptic holes is still adequate. Therefore, we will not discuss such a refinement.

The comparison between the analytical asymptotics and the numerical data for $\delta \mu$ is shown in Fig. 5 for the case $b_{x} / b_{y}=2$. As one can see, the droplet picture [Eq. (107), the left thin line in Fig. 15] remains accurate up to $n_{e} \sim 0.07 n_{1}$. The isolated depletion hole approximation [Eq. (114), the other thin line in Fig. 5 is accurate at $n_{e} \gtrsim 0.4 n_{1}$. We conclude that our analytical asymptotics, which are basically the perturbation theory results, indeed work at low and at high $n_{e}$, as expected.

\section{B. Trial ansatz method}

It is also aparent from Fig. 15 that the derived analytical formulas fail at intermediate $n_{e}$. For example, at the percolation threshold, $n_{p} \approx 0.31 n_{1}$, the actual value of $\delta \mu$ is about a factor of two off the nearest analytical asymptote. Going to higher orders in perturbation theory to reduce the discrepancy appears to be cumbersome and impracticable. It seems that the quantitatively accurate description of the ground state of the checkerboard model at intermediate $n_{e}$ is currently beyond the reach of controlled analytical methods.
There is however an alternative approach, idea of which was introduced in Ref. 11. Strictly speaking, this approach is uncontrolled yet it is semi-analytical and as we will show below, it reproduces the behavior of $\delta \mu$ at all $n_{e}$ remarkably well, both for the symmetric and for the asymmetric checkerboards. In its simplest implementation, this method amounts to adopting the following trial ansatz (TA) for the ground state density distribution:

$$
n_{a}(\mathbf{r})=\theta\left(\sigma-\sigma_{\mathrm{DR}}\right) \sqrt{\sigma^{2}(\mathbf{r})-\sigma_{\mathrm{DR}}^{2}} .
$$

Here $\theta(x)$ is the step-function and $\sigma_{\mathrm{DR}}$ is a constant that must obey the condition

$$
\int_{0}^{b_{x}} d x \int_{0}^{b_{y}} d y n_{a}(x, y)=n_{e} b_{x} b_{y} .
$$

Clearly, $n_{a}(\mathbf{r})$ is entirely fixed by $n_{e}$ and $\sigma(\mathbf{r})$ with no adjustable parameters. Why choosing the trial state in this form? Several reasons can be given. First, it is consistent with the notion that $n(\mathbf{r})$ is determined primarily by the behavior of $\sigma$ at points nearby. (After all, the interactions do decay with distance). Second, unless a function $\sigma(\mathbf{r})$ possesses multiple widely different lengthscales, the behavior of $\sigma$ in the vicinity of a given point $\mathbf{r}$ is dictated predominantly by the value of $\sigma(\mathbf{r})$ at the same point (e.g., small $\sigma$ tend to be located near minima, large $\sigma$ - near maxima). Consequently, the purely local ansatz $n(\mathbf{r})=n_{a}[\sigma(\mathbf{r})]$ seems reasonable. Third, Eq. (115) preserves the two asymptotic characteristics of the exact solution: a square-root singularity at the edges of the metallic regions (cf. Sec. I) and the perfect screening $n \rightarrow \sigma$ at large $n$ (cf. Sec. IIII). Fourth, one can verify that Eq. (115) is exact for the DR in the from of an infinite slit [Eq. (14)] and is also rather accurate for the round depletion hole [Eq. (12)].

Perhaps, the only serious deficiency of the proposed TA is the omission of the funneling effect of the saddle points. Indeed, according to Eq. (115) the boundaries of the DRs coincide with the $\sigma(\mathbf{r})=\sigma_{\mathrm{DR}}=$ const contour, whereas we showed in Sec. IV that there are logarithmic deviations from such a behavior, and these are noticeable in Figs. 3and 4 At any rate, the ansatz (115) is probably the simplest form that one can write down, so it makes sense to examine how it performs. Having learned its strengths and limitations, one will be in a better position to apply this kind of methods in situations where the brute force numerical simulations are difficult, such as in the models of disordered systems 16

The implementation of the TA method goes as follows. First one selects a reasonably dense set of $n_{e}$ and determines the corresponding $\sigma_{\mathrm{DR}}$ by solving Eq. (116) on the computer. In practice, we did it by approximating the integrals in Eq. (116) by a sum over the grid points. Then, for each $\sigma_{\mathrm{DR}}$, one evaluates the total energy energy of the corresponding trial state (115). Finally, the electrochemical potential $\delta \mu$ is computed by a numerical differentiation of the total energy with respect to $n_{e}$. The results of 

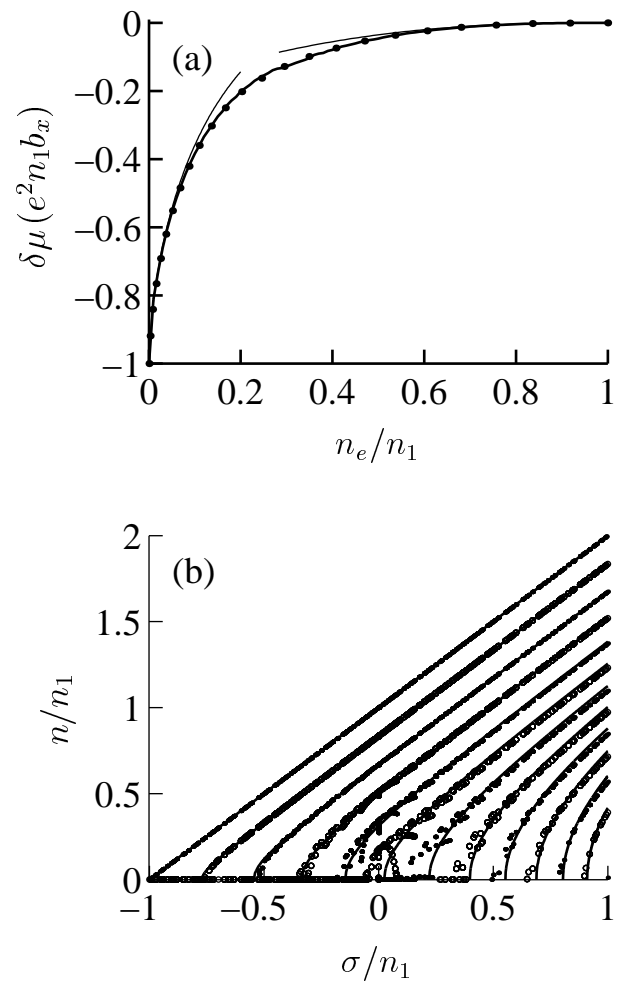

FIG. 6: Comparison between the TA method, analytical asymptotics, and numerical simulations for the symmetric checkerboard, $b_{x}=b_{y}$. (a) The correction $\delta \mu$ [cf. Eq. (7)] to the electrochemical potential as a function of $n_{e}$. The meaning of the lines and the dots is the same as in Fig. 4 (b) The scatter plot of $n$ vs. $\sigma$. Solid curves are the predictions of the TA for same densities $n_{e}$ as the dots at the top graph but skipping every other $n_{e}$ point for clarity. The leftmost curve is for $n_{e}=n_{1}$, the rightmost one (which degenerates into a single point) - for $n_{e}=0$. The symbols nearby each curve are from numerical simulations for the corresponding $n_{e}$.

such calculations are shown by the thick lines in Figs. 5 and 6(a). As one can see, the agreement between the TA method results for $\delta \mu$ and the corresponding numerical datapoints is very good.

To test the TA method further we can directly compare the density distribution $n_{a}(\mathbf{r})$ with the numerically determined ground state $n(\mathbf{r})$. Such a comparison is shown in Fig. 6 (b) where we present a scatter plot of $n$ vs. $\sigma$, for the case $b_{x}=b_{y}$. The spread of the symbols (numerical data) with respect to the the solid lines indicates that our TA is certainly not exact. However, such a spread is not dramatic, and so Eq. (115) is a viable approximation, especially at low and at high $n_{e}$.

One more quantity we can do the comparison for is the DR area fraction $f_{\mathrm{DR}}$. As one can see from Fig. 7 the TA method performs quite well at all $n_{e}$, while the analytical asymptotics [Eqs. (105) and (112)] are obeyed in their respective validity domains.

Finally, let us discuss the estimate of the percolation threshold that follows from the TA. According to the

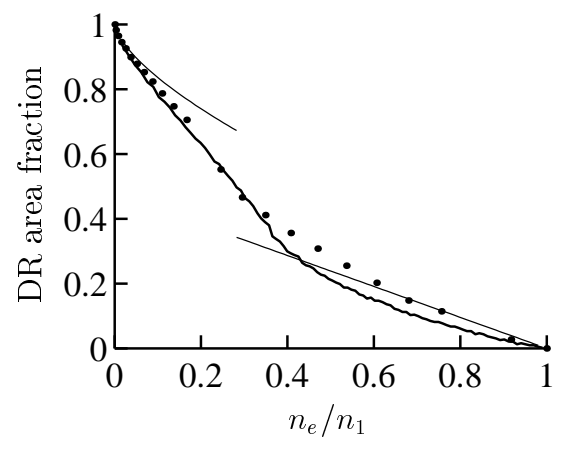

FIG. 7: Depleted area fraction according to the analytical asymptotics of Eqs. (105) and (112) (thin lines), numerical simulations (dots), and the TA method (thick line) for the symmetric checkerboard.

TA, the boundaries of the DR are defined by the equation $\sigma(\mathbf{r})=\sigma_{\mathrm{DR}}\left(n_{e}\right)$. Therefore, the percolation occurs at the average density $n_{p}^{*}$ that satisfies the relation $\sigma_{\mathrm{DR}}\left(n_{p}^{*}\right)=n_{p}^{*}$. Under this condition, the DR boundary passes simultaneously through all the saddle-points in the system. For example, within a single rectangular unit cell, the DR has the shape of a rhombus with vertices at the midpoints of the cell edges. Solving the above equation numerically, we found $n_{p}^{*} \approx 0.31 n_{1}$. This number is independent of $z_{c b}=b_{y} / b_{x}$ because checkerboards with different $z_{c b}$ can be mapped onto each other by rescaling the coordinate axes. Within the TA, such a rescaling does not change the topology of the DRs or the average electron density.

Clearly, the TA is unable to resolve the existence of two separate thresholds, $n_{p y}$ and $n_{p}$ in the asymmetric checherboard. Within the TA, the percolation occurs simultaneously in the $x$ - and $y$-directions. Still, $n_{p}^{*}$ is remarkably close to the upper (global) percolation threshold determined numerically for $z_{c b}=2$, see Eq. (97). This is also the case at larger $z_{c b}$, e.g., at $z_{c b}=3$ where we found $n_{p}=0.32 n_{1}$. On the other hand, at lower $z_{c b}$ the discrepancy grows and reaches its largest relative size of about $30 \%$ at $z_{c b}=1$, see Eq. (96). We believe that these discrepancies (one threshold instead of two and the value of $n_{p}$ ) originate from the two drawbacks of the TA method we mentioned earlier. One is its unability to handle widely separate lengthscales, which is the case in checkerboards with large $z_{c b}$. The other is its weakness in dealing with the saddle-points. The funneling effect of the saddle-points allows the electron droplets to reach them sooner as $n_{e}$ increases. Therefore, the continuity of the electron liquid is established at a lower $n_{e}$ compared to that predicted by the TA.

One may wonder why the TA method is able to predict $n_{p}$ with a much higher accuracy ${ }^{11}(\sim 10 \%)$ in the case of a random $\sigma(\mathbf{r})$. One possible explanation is as follows. The funneling effect of the saddle-points that is mishandled by the TA method is especially pronounced in the checkerboard geometry because all the saddle-points have 
the same value of $\sigma$, so that the percolation contour has to pass through all of them simultaneously. Thus, the inaccuracy of our TA is maximized precisely at $n=n_{p}$. In contrast, in the case of a random $\sigma(\mathbf{r})$ the percolation contour passes precisely through the center of a saddlepoint very rarely, and so the TA works very well.

We conclude that the TA method is an excellent and convenient tool for determining the ground-state energy and depleted area fraction but it may be less accurate when it comes to more subtle parameters of the real-space structure, especially if those are heavily dominated by the saddle-points or a hierarchy of multiple lengthscales.

\section{Acknowledgments}

This work is supported by Chris and Warren Hellman Fund and by Alfred P. Sloan Foundation. I am grateful to Sen Yang for participation in early stages of this project. I thank I. A. Larkin and B. I. Shklovskii for valuable comments on the manuscript.

\section{APPENDIX A: ELLIPSOIDAL HARMONICS}

The ellipsoidal harmonics ${ }^{27}$ of the first and the second kinds, $E_{m}^{p}(\xi)$ and $F_{m}^{p}(\xi)$, respectively, are defined as the two linearly independent solutions of the Lamé equation (for $\Lambda$ )

$$
f(\xi) \frac{d^{2} \Lambda}{d \xi^{2}}+\frac{1}{2} \frac{d f}{d \xi} \frac{d \Lambda}{d \xi}=\left[m(m+1) \xi^{2}-\left(1+k_{h}^{2}\right) p\right] \Lambda, \quad f(\xi) \equiv\left(\xi^{2}-1\right)\left(\xi^{2}-k_{h}^{2}\right) .
$$

For each $m$, which has to be a natural number, $p$ can take any of $2 m+1$ different values that depend on $k_{h}$. Functions $E_{m}^{p}(\lambda)$ and $F_{m}^{p}(\lambda)$ at $\lambda^{2} \geq 1$ are related by

$$
F_{m}^{p}(\lambda)=(2 m+1)\left|E_{m}^{p}(\lambda)\right| \int_{\lambda}^{\infty} \frac{d l}{\sqrt{f(l)}\left|E_{m}^{p}(l)\right|^{2}}
$$

At large $\lambda, F_{m}^{p}(\lambda) \propto 1 / \lambda^{m+1}$. As a rule, $F_{m}^{p}$ 's are not expressed in terms of elementary functions. For example, for $E_{m}^{p}$ given by Eqs. (41)-(42), Eq. (A2) leads to the following $F_{m}^{p}$ :

$$
\begin{aligned}
\frac{1}{3} F_{1}^{p_{1}}(\lambda) & =\frac{1}{1-k_{h}^{2}}\left[\frac{\sqrt{\lambda^{2}-k_{h}^{2}}}{\lambda}-\sqrt{\lambda^{2}-1} E\left(\arcsin \frac{1}{\lambda}, k_{h}\right)\right] \\
\frac{1}{7} F_{3}^{r_{ \pm}}(\lambda) & =\frac{\left(\lambda^{2}-C_{ \pm}\right) \sqrt{\lambda^{2}-k_{h}^{2}}}{\left(C_{ \pm}-1\right)^{2}\left(1-k_{h}^{2}\right)}+\sqrt{\lambda^{2}-1}\left(\lambda^{2}-C_{ \pm}\right)\left[A_{ \pm} E\left(\arcsin \frac{1}{\lambda}, k_{h}\right)-B_{ \pm} F\left(\arcsin \frac{1}{\lambda}, k_{h}\right)\right] \\
A_{ \pm} & =\frac{1-k_{h}^{2}+2 C_{ \pm}^{2}-2 C_{ \pm} k_{h}^{2}}{2 C_{ \pm}\left(C_{ \pm}-1\right)^{2}\left(k_{h}^{2}-C_{ \pm}\right)\left(1-k_{h}^{2}\right)}, \quad B_{ \pm}=\frac{1}{2 C_{ \pm}\left(C_{ \pm}-1\right)\left(k_{h}^{2}-C_{ \pm}\right)}
\end{aligned}
$$

where $E$ and $F$ are the elliptic integrals ${ }^{22}$ However, for $k_{h}=0$, these formulas simplify to

$$
\begin{aligned}
& \frac{1}{3} F_{1}^{p_{1}}(\lambda)=1-\arcsin (1 / \lambda) \\
& \frac{1}{7} F_{1}^{r_{+}}(\lambda)=\frac{5}{12}\left[15 \lambda^{2}-11-15\left(\lambda^{2}-\frac{2}{5}\right) \sqrt{\lambda^{2}-1} \arcsin (1 / \lambda)\right] \\
& \frac{1}{7} F_{1}^{r_{-}}(\lambda)=-\frac{1}{4 \lambda^{2}}-\frac{5}{8}+\frac{15}{8} \lambda^{2}-\frac{15}{8} \lambda^{2} \sqrt{\lambda^{2}-1} \arcsin (1 / \lambda)
\end{aligned}
$$

As an application of these formulas, one can derive the electrostatic potential $\Phi(\mathbf{r}, z)$ around the elliptic DR discussed in Sec. III To do so one needs to substitute Eqs. (A3) and (A4) for $F_{m}^{p}$, Eqs. (41) and (42) for $E_{m}^{p}$, and also Eqs. (52) and (53) for $\alpha_{m}^{p}$ into the series expansion (38). Combining such an expression for $\Phi(\mathbf{r}, z)$ with Eq. (60) one can then, in principle, deduce the formula for the density profile $n(\mathbf{r})$ of the electron liquid outside the DR. However, this calculation is not presented here because for a generic $k_{h}$ the result is rather unilluminating. The two notable exceptions are $k_{h}=0$, where one obtains a circular DR with $n(\mathbf{r})$ given by Eq. (12), and $k_{h}=1$ where the DR is an infinite depletion strip and Eq. (14) holds.
1 I. N. Sneddon, Mixed Boundary Value Problems in Potential Theory (North-Holland, Amsterdam, 1966).
${ }^{2}$ T. Deruelle, K. Ensslin, P. M. Petroff, A. L. Efros, and 
F. G. Pikus, Phys. Rev. B 45, 9082 (1992).

3 V. G. Burnett, A. L. Efros, and F. G. Pikus, Phys. Rev. B 48, 14365 (1993).

${ }^{4}$ V. J. Goldman and B. Su, Science 267, 1010 (1995).

${ }^{5}$ C. E. Nebel, J. Rogg, M. K. Kelly, B. Dahlheimer, M. Rother, M. Bichler, W. Wegscheider, and M. Stutzmann, J. Appl. Phys. 82, 1497 (1997).

${ }^{6}$ I. A. Larkin and V. B. Shikin, Phys. Lett. A 151, 9877 (1992).

7 D. B. Chklovskii, B. I. Shklovskii, and L. I. Glazman, Phys. Rev. B 46, 4026 (1992); ibid. 46, 15606 (E) (1992).

8 D. B. Chklovskii, K. A. Matveev, and B. I. Shklovskii, Phys. Rev. B 47, 12605 (1993).

9 M. M. Fogler, E. I. Levin, and B. I. Shklovskii, Phys. Rev. B 49, 13767 (1994).

10 J. H. Davies and I. A. Larkin, Phys. Rev. B 49, 4800 (1994).

11 M. M. Fogler, Phys. Rev. B (in press); cond-mat/0310010 (unpublished).

12 We assume that electron system contains only one type of charge carriers, say, electrons, so that the local density $e n(\mathbf{r})$ is nonpositive everywhere $(e=-|e|$ is the electron charge).

13 A. L. Efros and B. I. Shklovskii, Electronic Properties of Doped Semiconductors (Springer, New York, 1984).

14 V. A. Gergel' and R. A. Suris, Zh. Teor. Eksp. Fiz. 75, 1991 (1978) [Sov. Phys. JETP 48, 95 (1978)].

15 B. I. Shklovskii and A. L. Efros, Pis'ma Zh. Teor. Eksp. Fiz. 44, 520 (1986) [JETP Lett. 44, 669 (1986)].

16 A. L. Efros, F. G. Pikus, and V. G. Burnett, Phys. Rev. B 47, 2233 (1993).

17 Mathematically equivalent problem is treated in Sec. 10 of L. D. Landau and E. M. Lifshitz, Theory of Elasticity (Pergamon, Oxford, 1984).

18 L. D. Landau and E. M. Lifshitz, Electrodynamics of Continuous Media (Pergamon, Oxford, 1984).

19 H. A. Bethe, Phys. Rev. 66, 163 (1944).

20 See L. J. Walpole, Math. Proc. Camb. Phil. Soc. 67, 225 (1970) or the original work cited therein, F. W. Dyson, Quart. J. Math. Oxford Ser. 25, 259 (1891).

21 This proof is sketched in Ref. 1 without reference to Dyson.

22 I. S. Gradshteyn and I. M. Ryzhik Table of Integrals, Series, and Products, 6th ed., edited by A. Jeffrey and D. Zwillinger (Academic Press, San Diego, 2000).

23 Single Charge Tunneling: Coulomb Blockade Phenomena in Nanostructures, edited by H. Grabert and M. H. Devoret (Plenum, New York, 1992).

24 S. R. Patel, S. M. Cronenwett, D. R. Stewart, A. G. Huibers, C. M. Marcus, C. I. Duruöz, J. S. Harris, K. Campman, and A. C. Gossard, Phys. Rev. Lett. 80, 4522 (1998).

25 K.-H. Ahn, K. Richter, and I.-H. Lee, Phys. Rev. Lett. 83, 4144 (1999).

26 A. Cohen, K. Richter, and R. Berkovits, Phys. Rev. B 60, 2536 (1999).

27 W. E. Byerly, An Elementary Treatise on Fourier's Series, and Spherical, Cylindrical, and Ellipsoidal Harmonics, with Applications to Problems in Mathematical Physics
(Dover, New York, 1959).

28 For review, see E. Abrahams, S. V. Kravchenko, and M. P. Sarachik, Rev. Mod. Phys. 73, 251 (2001).

29 J. P. Eisenstein, L. N. Pfeiffer, and K. W. West, Phys. Rev. B 50, 1760 (1994).

30 S. C. Dultz and H. W. Jiang, Phys. Rev. Lett. 84, 4689 (2000); S. C. Dultz, B. Alavi, and H. W. Jiang, condmat/0210584 (unpublished).

31 S. Ilani, A. Yacoby, D. Mahalu, and H. Shtrikman, Phys. Rev. Lett. 84, 3133 (2000); Science 292, 1354 (2001).

32 S. Ilani, J. Martin, E. Teitelbaum, J. H. Smet, D. Mahalu, and A. Yacoby, Nature (London) 427, 328 (2004).

33 B. J. van Wees, H. van Houten, C. W. J. Beenakker, J. G. Williamson, L. P. Kouwenhoven, D. van der Marel, and C. T. Foxon, Phys. Rev. Lett. 60, 848 (1988); D. A. Wharam, T. J. Thornton, R. Newbury, M. Pepper, H. Ahmed, J. E. F. Frost, D. G. Hasko, D. C. Peacock, D. A. Ritchie, and G. A. C. Jones, J. Phys. C 21, L209 (1988).

34 O. A. Tkachenko, V. A. Tkachenko, D. G. Baksheyev, K. S. Pyshkin, R. H. Harrell, E. H. Linfield, D. A. Ritchie, and C. J. B. Ford, J. Appl. Phys. 89, 4993 (2001).

35 M. Büttiker, Phys. Rev. B 41, 7906 (1990).

36 H. A. Fertig and B. I. Halperin, Phys. Rev. B 36, 7969 (1987).

37 J. E. F. Frost, K.-F. Berggren, M. Pepper, M. Grimshaw, D. A. Ritchie, A. C. Churchill, and G. A. C. Jones, Phys. Rev. B 49, 11500 (1994).

38 A. Szafer and A. D. Stone, Phys. Rev. Lett. 62, 300 (1989).

39 M. Yosefin and M. Kaveh, Phys. Rev. Lett. 64, 2819 (1990)

40 S. He and S. Das Sarma, Phys. Rev. B 48, 4629 (1993).

41 F. A. Maao, I. V. Zozulenko, and E. H. Hauge, Phys. Rev. B 50, 17320 (1994).

42 L. I. Glazman, G. B. Lesovik, D. E. Khmel'nitskii, and R. I. Shekhter, Pis'ma Zh. Eksp. Teor. Fiz. 48, 238 (1988) [JETP Lett. 48, 238 (1988)]; A. Yacoby and Y. Imry, Phys. Rev. B 41, 5341 (1990).

43 As already explained above, in a physical system there is an additional small-distance cutoff equal to $a_{\mathrm{e}-\mathrm{e}}(x)$.

44 J. D. Jackson, Classical Electrodynamics, 2nd ed. (Wiley, New York, 1975).

45 The renormalized value $U_{y y}^{\text {ren }} \approx 3.60$ also needed here was found by fitting numerical $\Phi(0, y)$ to a parabola. As expected, $U_{y y}^{\text {ren }}$ is a bit larger than its perturbative estimate $U_{y y}^{\text {ren }}=U_{y y}-\left(U_{x x} / 2\right)=3.5$.

46 G. Eytan, Y. Yayon, M. Rappaport, H. Shtrikman, and I. Bar-Joseph, Phys. Rev. Lett. 81, 1666 (1998).

47 B. J. LeRoy, M. A. Topinka, R. M. Westervelt, K. D. Maranowski, and A. C. Gossard, Appl. Phys. Lett. 80, 4431 (2002).

48 A. Bachtold, M. S. Fuhrer, S. Plyasunov, M. Forero, E. H. Anderson, A. Zettl, and P. L. McEuen, Phys. Rev. Lett. 84, 6082 (2000).

${ }^{49}$ For the exception of the $b_{x}=\infty$ case, where the problem becomes one-dimensional, see Ref. 10 . 